\title{
Crystal Plasticity Finite Element Modeling of Discrete Twin Evolution in Polycrystalline Magnesium
}

\author{
Jiahao Cheng ${ }^{\mathrm{a}, 1}$, Somnath Ghosh ${ }^{\mathrm{b}, 2, *}$ \\ ${ }^{a}$ Department of Civil Engineering \\ ${ }^{b}$ Departments of Civil and Mechanical Engineering, Johns Hopkins University, Baltimore, MD 21218
}

\begin{abstract}
This paper develops an advanced, image-based crystal plasticity finite element (CPFE) model, for predicting explicit twin formation and associated heterogeneous deformation in single crystal and polycrystalline microstructures of hexagonal close-packed or hcp materials, such as magnesium. Twin formation is responsible for premature failure of many hcp materials. The physics of nucleation, propagation and growth of explicit twins are considered in the CPFE formulation. The twin nucleation model is based on dissociation of sessile dislocations into stable twin loops, while propagation is assumed by atoms shearing on twin planes and shuffling to reduce the thermal activation energy barrier. The explicit twin evolution model however has intrinsic issues of low computational efficiency. Very fine simulation time steps with enormous computation costs are required to simulate the fast propagating twin bands and associated strain localization. To improve the computational efficiency, a multi-time scale subcycling algorithm is developed. It decomposes the computational domain into sub-domains of localized twins requiring very fine timesteps and complementary domains of relatively low resolution. Each sub-domain updates the stress and the deformation-dependent variables in different rates, followed by a coupling at the end of every coarse time step to satisfy global equilibrium. A 6-fold increase in computing speed is obtained for a polycrystalline $\mathrm{Mg}$ microstructure simulation in this paper. CPFE simulations of high purity $\mathrm{Mg}$ microstructures are compared with experiments with very good agreement in stress-strain response as well as heterogeneous twin formation with strain localization.
\end{abstract}

Keywords: Explicit Twin Evolution, Crystal Plasticity Finite Element, Magnesium,

\footnotetext{
${ }^{*}$ Corresponding author: Tel: +1-410-516-7833; fax: +1-410-516-7473

Email address: sghosh20@ jhu .edu (Somnath Ghosh )

${ }^{1}$ Graduate Research Associate

${ }^{2}$ M. G. Callas Professor
} 
Subcycling, Tension-Compression Asymmetry

\section{Introduction}

Metals and alloys with low-symmetry hexagonal closed packed ( $h c p$ ) crystallographic structure, like $\mathrm{Mg}$ and $\mathrm{Mg}$ alloys, consist of 5 different families of slip systems. They are the basal $\langle a\rangle$, prismatic $\langle a\rangle$, pyramidal $\langle a\rangle$, first order pyramidal $\langle c+a\rangle$ and second order pyramidal $\langle c+a\rangle$, with a total of 30 slip systems. The critical resolved shear stress (CRSS) for basal $\langle a\rangle$ slip systems is much lower than that for prismatic $\langle a\rangle$ or pyramidal $\langle a\rangle$ and $\langle c+a\rangle$ slip systems, especially at lower temperatures. The large differences in CRSS and lack of dislocation glide on the pyramidal systems introduce pronounced plastic anisotropy and tension-compression asymmetry in their mechanical response. It is impossible for these low symmetry $h c p$ grains, constrained by their neighbors, to plastically deform by basal slip alone. Both non-basal $\langle c+a\rangle$ slip and twinning can accommodate deformation normal to the basal plane. However, the high $\langle c+a\rangle$ slip system CRSS in comparison with twinning stresses result in significant localized deformation twinning in polycrystalline $h c p$ microstructures. A schematic of slip and twin systems in $h c p$ crystals is shown in figure 1. Discrete micro-twin bands are observed in the micrograph of figure 2c. Twinning causes sharp reorientation in the crystallographic lattice structure. This leads to strain localization and subsequent fracture inside the twin bands (Yu et al., 2011).

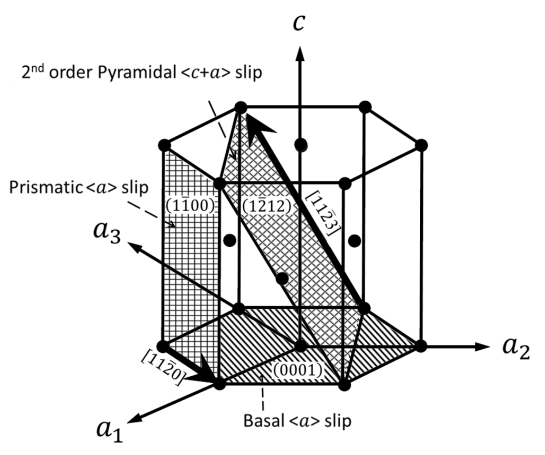

(a)

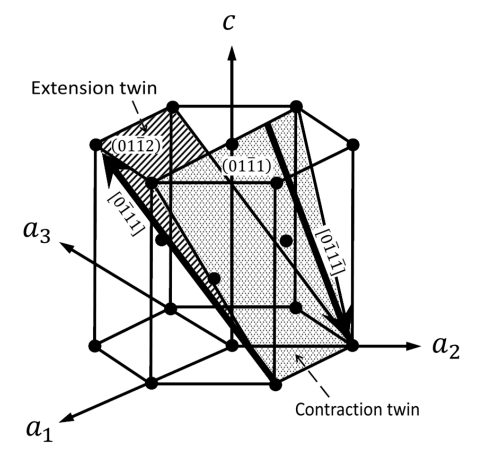

(b)

Figure 1: Schematic diagrams showing: (a) active slip systems and (b) twin systems, in hcp magnesium. 


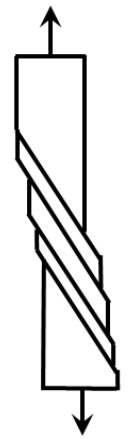

(a)

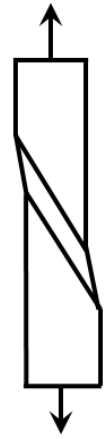

(b)

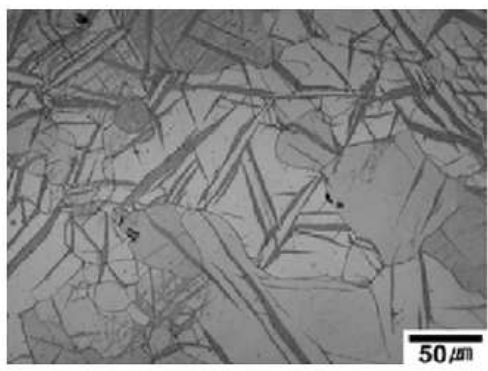

(c)

Figure 2: Schematic of shear deformation due to: (a) dislocation slip and (b) deformation twinning; and (c) optical microstructure showing deformation twins in tension test of a rolled AZ31 $\mathrm{Mg}$ alloy at $4 \%$ strain (from (Hong et al., 2010)).

Since plastic flow inhomogeneities are intricately dependent on the morphological and crystallographic characteristics of the microstructure, it is important to develop imagebased micro-mechanical models of deformation and twinning. Crystal plasticity finite element (CPFE) models have been implemented to model deformation and twinning in Mg in (Staroselsky and Anand, 2003a; Graff et al., 2007; Izadbakhsh et al., 2011, 2012; Zhang and Joshi, 2012; Abdolvand and Daymond, 2013a). Most of the twinning models for CPFE simulations adopt a volume-fraction based approach to represent twinning in the microstructure. In this representation, sometimes termed as the pseudo-slip approach, the diffused twin volume fraction evolution and lattice slip are evaluated simultaneously at integration points. The morphological identity of the discrete twin bands and heterogeneity of the twinned microstructure is lost as a result. These models are generally not capable of predicting localization triggered by heterogeneous micro-twins. Recently, explicit twin formation models have been developed in CPFEM in (Abdolvand and Daymond, 2013b; Ardeljan et al., 2015) by incorporating phenomenological twin formation criteria and adaptive mesh generation methods. A thermodynamically consistent constitutive model has been developed from micromechanics consideration, accounting for the interaction between explicit twins and the matrix regions in (Mareau and Daymond, 2016). Explicit twin formation models accounting for physics-based nucleation, e.g. in (Cheng and Ghosh, 2015), propagation and interaction, are needed to accurately predict twin induced material failure. Another shortcoming with many of the twinning models using the CPFE framework is that they ignore the effect of geometrically necessary dislocations (GNDs). Dislocation density based CPFE models involving the evolution of GNDs and 
SSDs have been proposed and implemented in (Ma et al., 2006; Wong et al., 2016). These models account for size-effect due to GNDs in displacement-based CPFE models and incorporate displacement boundary conditions only. Robust strain gradient crystal plasticity theories, incorporating the storage of GNDs due to strain gradients, have been proposed in (Cermelli and Gurtin, 2001; Gurtin, 2006; Gurtin and Anand, 2009; Gurtin et al., 2010). These strain gradient crystal plasticity theories require the implementation of higher order boundary conditions corresponding to the primary solution fields of plastic strain or plastic strain rate. Although more accurate, the higher order theories have increased complexity of modeling and reduced efficiency due to additional degrees of freedom compared to displacement based CPFE formulations.

A significant obstacle to conducting high fidelity image-based CPFE simulations with explicit twin formation is its very low computational efficiency. The time-scale of crystallographic slip induced deformation is significantly higher than that of twin evolution inside twin-bands. Furthermore, localized deformation inside twin bands can cause numerical instabilities with the stiff crystal plasticity constitutive equations. These need very small numerical time-steps in the incremental solution process, which makes high resolution CPFE simulation of polycrystalline microstructures computationally prohibitive.

Various numerical methods have been proposed for improving the efficiency of stable time integration of crystal plasticity constitutive relations. These methods include judicious selection of appropriate starting values for iterative implicit integration scheme (Roters et al., 2010a), adding line-search to Newton-Raphson iterations (Ling et al., 2005), or explicit forward Euler time integration schemes (Rossiter et al., 2010). In (Li and Yang, 2012), an explicit algorithm is developed by introducing Taylor series expansion to recast the non-linear crystal plasticity equations into a set of linear equations with increments of stress and slip system resistances, which are solved by a two-level Gauss elimination procedure. Adaptive sub-stepping methods in (Sloan, 1987; Zhang et al., 2014) integrate the rate-dependent crystal plasticity models by splitting the deformation increment into sub-steps with error control. These methods are however not appropriate for non-local models of micro-twinning. Subcycling induced time-integration algorithms have been proposed in (Belytschko and Mullen, 1977; Smolinski et al., 1988; Neal and Belytschko, 1989; Smolinski, 1996; Smolinski and Wu, 1998), where different time-steps are used in different parts of the computational domain. This method has been mainly used for explicit finite element analysis of structures, in which locally-variant time steps are chosen according to the local stability requirements of the subdomain.

This paper develops an image-based crystal plasticity FE model for dislocation-mediated 
deformation in polycrystalline pure $\mathrm{Mg}$ with explicit depiction of nucleation, propagation, and growth of twins. Hardness evolution laws for statistically-stored and geometrically necessary dislocations are developed to be consistent with lower order theories in (Ma et al., 2006; Roters et al., 2010a). Physics-based criteria and models for twin nucleation and growth are developed within the CPFE framework. A subcycling method, based on partitioning of the microstructural domain containing twins, is implemented to enable discrete twin manifestation by overcoming the low computational efficiency of CPFE simulations. Time steps in the twinned regions are much smaller that those in the exterior regions in this algorithm. This paper is organized as follows. The crystal plasticity framework accounting for crystallographic slips and deformation twinning is presented in section 2 2. Physics-based models for explicit twin nucleation and growth are developed in section 3. Numerical implementation of the the model and time integration schemes are discussed in section 4 and the adaptive subcycling method for twin formation is developed and validated in section 5. Finally the CPFE model is used for validation studies, with respect to characteristics of twin evolution and overall response, of pure $\mathrm{Mg}$ polycrystals in section 6. The model is summarized in section 7 .

\section{Crystal Plasticity Model due to Crystallographic Slip and Twinning}

Deformation in single and polycrystalline metals and alloys are conventionally simulated using crystal plasticity finite element (CPFE) models. Crystal plasticity constitutive models account for dislocation glide and twinning on crystallographic slip and twin systems. A significant body of work exists on micromechanical modeling using crystal plasticity models due to glide on slip systems in (Staroselsky and Anand, 2003b; Busso et al., 2000; Zambaldi et al., 2007; Roters et al., 2010b; Hasija et al., 2003; Deka et al., 2006; Venkataramani et al., 2007), using power law description (Asaro and Needleman, 1985 ) and the thermally activated theory of plastic flow by (Kocks et al., 1975). A crystal plasticity constitutive model for plastic deformation of pure $\mathrm{Mg}$ and the $\mathrm{Mg}$ alloy AZ31 has been proposed in (Cheng and Ghosh, 2015). The flow rule and the twin nucleation model used in this paper are the same as described in (Cheng and Ghosh, 2015). The evolution laws for statistically-stored and geometrically necessary dislocations have been altered from those in (Cheng and Ghosh, 2015) to be consistent with dislocation evolution theories. Novel deformation twin propagation and growth models are developed in this paper with the goal of explicitly tracking individual twins in the ensemble.

The total deformation gradient $\mathbf{F}$ is multiplicatively decomposed into a component $\mathbf{F}^{e}$ that accounts for elastic stretching and rigid-body rotation of the crystal, and a component 
$\mathbf{F}^{p}$ associated with the incompressible plastic flow, given as:

$$
\mathbf{F}=\mathbf{F}^{e} \mathbf{F}^{p}
$$

The stress-strain relation is written in the elastically deformed reference configuration, in terms of the second Piola-Kirchoff stress $\mathbf{S}$ and the elastic Green-Lagrange strain tensor $\mathbf{E}^{e}$ as:

$$
\mathbf{S}=\mathbb{C}^{e}: \mathbf{E}^{e}
$$

where $\mathbb{C}^{e}$ is a fourth-order anisotropic elasticity tensor. Plastic flow is the result of dislocationslip as well as twin evolution in this model. In the absence of twinning, plastic deformation is expressed in terms of the plastic velocity gradient $\mathbf{L}^{p}$ as:

$$
\mathbf{L}^{p}=\dot{\mathbf{F}}^{p} \mathbf{F}^{p-1}=\sum_{\alpha=1}^{N_{s l i p}} \dot{\gamma}^{\alpha} \mathbf{s}_{0, s l i p}^{\alpha}
$$

where $\dot{\gamma}^{\alpha}$ is the slip rate on a slip system $\alpha$ and $N_{\text {slip }}$ is the total number of slip systems. The Schmid tensor associated with $\alpha$-th slip system $\mathbf{s}_{0, s l i p}^{\alpha}$ is expressed in terms of the slip direction $\mathbf{m}_{0, \text { slip }}^{\alpha}$ and slip plane normal $\mathbf{n}_{0, \text { slip }}^{\alpha}$ in the reference configuration, i.e. $\mathbf{s}_{0, s l i p}^{\alpha}=\mathbf{m}_{0, s l i p}^{\alpha} \otimes \mathbf{n}_{0, s l i p}^{\alpha}$.

Twinning reorients the crystallographic lattice symmetrically by reflection across a mirror or twin plane in the reference configuration. In the twinned region, the plastic flow takes place by gliding of twin partial dislocations on twin planes, as well as by dislocation glide. The plastic velocity gradient in twinned region is expressed as:

$$
\mathbf{L}^{p}=\dot{\mathbf{F}}^{p} \mathbf{F}^{p-1}=\sum_{\beta=1}^{N_{t w i n}} \dot{\gamma}_{t w}^{\beta} \mathbf{s}_{0, t w}^{\beta}+\sum_{\alpha=1}^{N_{\text {slip }}} \dot{\tilde{\gamma}}^{\alpha} \tilde{\mathbf{s}}_{0, s l i p}^{\alpha}
$$

where $\dot{\gamma}_{t w}^{\beta}$ is the shearing rate on a twin system $\beta$ and $N_{\text {twin }}$ is the total number of twin systems in the crystal. The Schmid tensor $\mathbf{s}_{0, t w}^{\beta}$ of a twin system $\beta$ is expressed in terms of the twin shearing direction vector $\mathbf{m}_{0, t w}^{\beta}$ and the twin plane normal $\mathbf{n}_{0, t w}^{\beta}$ in the reference configuration, i.e. $\mathbf{s}_{0, s l i p}^{\beta}=\mathbf{m}_{0, t w}^{\beta} \otimes \mathbf{n}_{0, t w}^{\beta}$. Dislocation slip in the twinned volume occurs on a slip plane $\tilde{\mathbf{n}}_{0, s l i p}^{\alpha}$ in the slip direction $\tilde{\mathbf{m}}_{0, \text { slip }}^{\alpha}$, with mirror-symmetry to the directions $\mathbf{n}_{0, s l i p}^{\alpha}$ and $\mathbf{m}_{0, s l i p}^{\alpha}$ in the matrix region by the following relations:

$$
\tilde{\mathbf{m}}_{0, \text { slip }}^{\alpha}=\mathbf{C}^{\beta} \mathbf{m}_{0, s l i p}^{\alpha} \quad \widetilde{\mathbf{n}}_{0, \text { slip }}^{\alpha}=\mathbf{C}^{\beta^{-T}} \mathbf{n}_{0, \text { slip }}^{\alpha}
$$

where $\mathbf{C}^{\beta}$ is the re-orientation matrix of the twin system $\beta$, given in (Niewczas, 2010). In equation (4) the Schmid factor of the re-orientated slip system $\alpha$ in the twinned region is 
expressed as $\tilde{\mathbf{s}}_{0, \text { slip }}^{\alpha}=\tilde{\mathbf{m}}_{0, \text { slip }}^{\alpha} \otimes \tilde{\mathbf{n}}_{0, \text { slip }}^{\alpha} \cdot \dot{\tilde{\gamma}}^{\alpha}$ is the slip rate in the re-orientated slip system $\alpha$.

The power law form of the flow rule in (Cheng and Ghosh, 2015), derived from the thermal activated dislocation glide model in (Kocks et al., 1975), is used for the slip rate on regular slip system $\alpha$ in $h c p$ materials. It is given as:

$$
\dot{\gamma}^{\alpha}=\dot{\gamma}_{0}^{\alpha}\left|\frac{\tau^{\alpha}-s_{a}^{\alpha}}{s_{*}^{\alpha}}\right|^{\frac{1}{m}} \operatorname{sign}\left(\tau^{\alpha}-s_{a}^{\alpha}\right)
$$

where $\dot{\gamma}_{0}^{\alpha}$ is a reference slip rate for slip system $\alpha$ and $m$ is the power law exponent representing strain-rate sensitivity. The resolved shear stress on slip system $\alpha$ is expressed as $\tau^{\alpha}=\mathbf{F}^{e T} \mathbf{F}^{e} \mathbf{S}: \mathbf{s}_{0}^{\alpha}$. The term $s_{a}^{\alpha}$ in the numerator is the athermal shear resistance due to the interaction of the stress-field between parallel dislocation lines or from grain boundaries and $s_{*}^{\alpha}$ is the thermal shear resistance due to local repelling obstacles, such as forest dislocations and dislocation jogs. An effective resolved shear stress is defined as $\tau_{\text {eff }}^{\alpha}=\tau^{\alpha}-s_{a}^{\alpha}$. Slip evolution on the re-orientated system in the twinned region is modeled in the same way as regular slip in the matrix. Therefore, the slip rate in equation (6) and the accompanying hardening model in section 2.1 also apply to the twinned region. The symbol ${ }^{\sim}$ will hence be omitted for simplicity.

\subsection{Evolution of slip system resistances}

The athermal and thermal shear resistances in equation (6) and their evolution are derived from dislocation-based mechanisms, and are consistent with the dislocation densitybased formulations given in (Ma et al., 2006). Two types of dislocations are considered in the evolution of both the athermal $\left(s_{a}^{\alpha}\right)$ and thermal $\left(s_{*}^{\alpha}\right)$ shear resistances. They are: (i) the statistically stored dislocations (SSDs) and (ii) the geometrically necessary dislocations (GNDs) (Ashby, 1970; Ma et al., 2006). SSDs are associated with the homogeneous components of plastic flow and are characterized by a vanishing net Burgers vector in the microstructure. On the other hand, GNDs correspond to stored polarized dislocation densities and are related to the curl of plastic deformation gradient in the intermediate (elastically unloaded) configuration. GND accumulation is necessary for accommodating crystal lattice curvatures in single crystal bending or near grain boundaries of polycrystalline aggregates. Detailed derivation of the hardening model is provided in the Appendix A. The resulting athermal and thermal hardening rates due to the evolution of SSDs are given as:

$$
\begin{aligned}
& \dot{s}_{a, S S D}^{\alpha}=h_{a}^{\alpha \beta}\left|\dot{\gamma}^{\beta} \sin \left(\boldsymbol{n}_{\mathbf{0}}{ }^{\alpha}, \boldsymbol{t}_{\mathbf{0}}{ }^{\beta}\right)\right| \\
& \dot{s}_{*, S S D}^{\alpha}=h_{*}^{\alpha \beta}\left|\dot{\gamma}^{\beta} \cos \left(\boldsymbol{n}_{\mathbf{0}}{ }^{\alpha}, \boldsymbol{t}_{\mathbf{0}}{ }^{\beta}\right)\right|
\end{aligned}
$$


where the coefficient matrices $h_{a}^{\alpha \beta}$ and $h_{*}^{\alpha \beta}$ represent the hardening of athermal and thermal shear resistances on the slip system $\alpha$ due to activity on slip system $\beta$. These matrices are derived to be:

$$
\begin{array}{l|l}
h_{a}^{\alpha \beta}=q^{\alpha \beta} h_{a, r e f}^{\beta}\left|1-\frac{s_{a, S S D}^{\beta}}{s_{a, s a t}^{\beta}}\right|^{r} \operatorname{sign}\left(1-\frac{s_{a, S S D}^{\beta}}{s_{a, s a t}^{\beta}}\right) & (\text { no sum on } \beta) \\
h_{*}^{\alpha \beta}=q^{\alpha \beta} h_{*, r e f}^{\beta}\left|1-\frac{s_{*, S S D}^{\beta}}{s_{*, \text { sat }}^{\beta}}\right|^{r} \operatorname{sign}\left(1-\frac{s_{*, S S D}^{\beta}}{s_{*, \text { sat }}^{\beta}}\right) & (\text { no sum on } \beta)
\end{array}
$$

where $s_{a, s a t}^{\alpha}$ and $s_{*, s a t}^{\alpha}$ are the athermal and thermal saturation stresses for hardening caused by the SSD population. The exponent $r$ is a material constant, while $h_{a, r e f}$ and $h_{*, r e f}$ are respectively the reference hardening rates for athermal and thermal slip resistances, and $q^{\alpha \beta}$ is a matrix describing latent hardening.

The GND contributions to the slip system hardening are derived from two sources, viz. (i) dislocation components $\rho_{G N D, P}^{\alpha}$ parallel to the slip plane $\alpha$ that cause hardening due to the athermal shear resistance $s_{a}^{\alpha}$, and (ii) forest dislocation components $\rho_{G N D, F}^{\alpha}$, which contribute to hardening due to thermal shear resistance $s_{*}^{\alpha}$. These are given as:

$$
\begin{gathered}
s_{a, G N D}^{\alpha}=c_{1} G b \sqrt{\rho_{P, G N D}^{\alpha}} \\
s_{*, G N D}^{\alpha}=\frac{Q_{s l i p}}{c_{2} c_{3} b^{2}} \sqrt{\rho_{F, G N D}^{\alpha}}
\end{gathered}
$$

where $G$ is the shear modulus, $Q_{\text {slip }}^{\alpha}$ is the effective activation energy for dislocation slip and $c_{1}, c_{2}, c_{3}$ are constants representing the passing stress, jump-width, and obstaclewidth, respectively. GND accumulation leads to a closure failure of the Burgers circuit in the intermediate configuration of the crystal lattice. This can be measured in terms of the curl of the plastic deformation gradient per unit area in the reference configuration, which corresponds to the Nye's dislocation density tensor $\Lambda=-\left(\nabla_{X} \times \mathbf{F}^{p^{T}}\right)^{T}$ (Dai, 1997). The relation between the Nye's tensor and GND density components on each slip system is expressed as (Cheng and Ghosh, 2015):

$$
\boldsymbol{\Lambda}=\sum_{\alpha=1}^{n s l i p} \rho_{G N D, s}^{\alpha} \mathbf{b}_{\mathbf{0}}{ }^{\alpha} \otimes \mathbf{m}_{\mathbf{0}}{ }^{\alpha}+\rho_{G N D, e t}^{\alpha} \mathbf{b}_{\mathbf{0}}{ }^{\alpha} \otimes \mathbf{t}_{\mathbf{0}}{ }^{\alpha}+\rho_{G N D, e n}^{\alpha} \mathbf{b}_{\mathbf{0}}{ }^{\alpha} \otimes \mathbf{n}_{\mathbf{0}}{ }^{\alpha}
$$

where $\boldsymbol{\rho}_{G N D, s}, \boldsymbol{\rho}_{G N D, e t}$ and $\boldsymbol{\rho}_{G N D, e n}$ are the GND density components with screw, in-slipplane edge and normal-to-slip-plane edge characteristics, per unit volume in the reference configuration. $\mathbf{t}^{\alpha}$ is the tangent vector of dislocation line expressed as $\mathbf{t}_{0}^{\alpha}=\mathbf{m}_{0}^{\alpha} \times \mathbf{n}_{0}^{\alpha}$, 
and $\mathbf{b}_{0}^{\alpha}$ is the Burgers vector for a slip system $\alpha$ in the reference configuration. For $h c p$ crystals, there are more slip systems than the number of components in $\Lambda$ resulting in an under-determined system of linear equations (10). To overcome this, the solutions of $\rho_{G N D, s}, \rho_{G N D, e t}$ and $\rho_{G N D, e n}$ are obtained by solving a constrained minimization problem of minimizing the $L_{2}$ norm of the GND densities subject to the constraint equation (10) as (Anahid et al., 2011):

$$
\rho_{G N D}=\operatorname{Arg}\left[\operatorname{Min}\left\{\left\{\rho_{G N D}\right\}^{T}\left\{\rho_{G N D}\right\}+\{\boldsymbol{\lambda}\}^{T}\left([\mathbf{A}]\left\{\rho_{G N D}\right\}-\{\hat{\boldsymbol{\Lambda}}\}\right)\right\}\right]
$$

where $\left\{\boldsymbol{\rho}_{G N D}\right\}$ is a $36 \times 1$ vector column of GND components, $\{\boldsymbol{\lambda}\}$ is a $9 \times 1$ column vector containing components of the Lagrange multipliers, $\{\hat{\Lambda}\}$ is $9 \times 1$ vector form of the Nye's dislocation density tensor $\boldsymbol{\Lambda}$, and $[\mathbf{A}]$ is a $9 \times 36$ linear operator matrix containing the basis tensors $\mathbf{b}_{0}{ }^{\alpha} \otimes \mathbf{m}_{0}{ }^{\alpha}, \mathbf{b}_{0}{ }^{\alpha} \otimes \mathbf{t}_{0}{ }^{\alpha}$ and $\mathbf{b}_{0}{ }^{\alpha} \otimes \mathbf{n}_{0}{ }^{\alpha}$.

The screw and edge GND components $\rho_{G N D, s}, \rho_{G N D, e t}$ and $\rho_{G N D, e n}$ on each slip system contribute respectively to the parallel $\left(\rho_{G N D, P}^{\alpha}\right)$ and forest $\left(\rho_{G N D, F}^{\alpha}\right)$ components of GNDs, according to the relations:

$$
\begin{aligned}
\rho_{G N D, P}^{\alpha}= & \sum_{\beta=1}^{N_{\text {slip }}} \chi^{\alpha \beta}\left[\left|\rho_{G N D s}^{\beta} \sin \left(\mathbf{n}_{0}^{\alpha}, \mathbf{m}_{0}^{\beta}\right)\right|+\left|\rho_{G N D e t}^{\beta} \sin \left(\mathbf{n}_{0}^{\alpha}, \mathbf{t}_{0}^{\beta}\right)\right|\right. \\
& \left.+\left|\rho_{G N D e n}^{\beta} \sin \left(\mathbf{n}_{0}^{\alpha}, \mathbf{n}_{0}^{\beta}\right)\right|\right] \\
\rho_{G N D, F}^{\alpha}= & \sum_{\beta=1}^{N_{\text {slip }}} \chi^{\alpha \beta}\left[\left|\rho_{G N D s}^{\beta} \cos \left(\mathbf{n}_{0}^{\alpha}, \mathbf{m}_{0}^{\beta}\right)\right|+\left|\rho_{G N D e t}^{\beta} \cos \left(\mathbf{n}_{0}^{\alpha}, \mathbf{t}_{0}^{\beta}\right)\right|\right. \\
& \left.+\left|\rho_{G N D e n}^{\beta} \cos \left(\mathbf{n}_{0}^{\alpha}, \mathbf{n}_{0}^{\beta}\right)\right|\right]
\end{aligned}
$$

where $\chi_{\alpha \beta}$ is a matrix of coefficients that describes the interaction strength between different slip systems. The total athermal and thermal resistances may be expressed as the sum of two parts (see Appendix A for details). One is related to the evolving dislocation structure and the other is related to defects such as Peierls resistance, impurities and point defects etc., stated as:

$$
\begin{aligned}
& s_{a}^{\alpha}=s_{a, 0}^{\alpha}+\sqrt{\left(s_{a, S S D}^{\alpha}\right)^{2}+\left(s_{a, G N D}^{\alpha}\right)^{2}} \\
& s_{*}^{\alpha}=s_{*, 0}^{\alpha}+\sqrt{\left(s_{*, S S D}^{\alpha}\right)^{2}+\left(s_{*, G N D}^{\alpha}\right)^{2}}
\end{aligned}
$$

Here $s_{a, 0}$ and $s_{*, 0}$ are initial resistances that do not depend on the evolution of the dislocation structure. Experimental results for $h c p$ alloys, e.g. the titanium alloy Ti-6242 in 
(Cao et al., 2000; Semiatin and Bieler, 2001) and magnesium alloy NZ30K in (Li et al., 2013), have suggested that the initial yield stress at the onset of plastic flow is sensitive to the grain size. A Hall-Petch type relation has been proposed in (Cao et al., 2000; Semiatin and Bieler, 2001; Venkataramani et al., 2007) to augment the initial thermal shear resistance $\hat{s}_{*, 0}^{\alpha}$, based on the premise that mobile dislocation loops can propagate at applied stresses below the critical resolved shear stress (CRSS) for low initial dislocation densities. These dislocations pile up at grain boundaries, inducing stress concentrations to augment the plastic flow. More dislocation loops can pile up in larger grains leading to lower CRSS. Correspondingly, the initial shear resistance $\hat{s}_{*, 0}^{\alpha}$ is augmented with a grain size dependent term as:

$$
s_{*, 0}^{\alpha}=\hat{s}_{*, 0}^{\alpha}+\frac{K^{\alpha}}{\sqrt{D_{g}}}
$$

where $D_{g}$ is the equivalent grain diameter and the parameter $K^{\alpha}=\sqrt{\frac{(2-\nu) \pi \tau^{*} G b^{\alpha}}{2(1-\nu)}}$, in which $\tau^{*}$ is the barrier strength, taken as $\tau^{*}=0.01 G$. In addition to this grain-size dependence, the non-local GND model introduces a length-scale dependence into the athermal and thermal hardening rates. For deformation in polycrystalline aggregates, the accumulated GND densities increase with reduction in grain-size.

The crystal plasticity constitutive parameters for slip in pure $\mathrm{Mg}$ are calibrated following a process discussed in (Cheng and Ghosh, 2015). The calibrated parameters are listed in table 1 .

\section{Models for Explicit Twin Nucleation and Growth}

Deformation twinning leads to anisotropic plasticity, tension-compression asymmetry and premature failure of $\mathrm{Mg}$ as described in (Bettles and Gibson, 2005; Kainer, 2003; Barnett, 2007; Yu et al., 2011). The twin nucleation model developed in (Cheng and Ghosh, 2015) is summarized in section 3.1. Subsequently, section 3.2 proposes a novel twin growth model and its numerical implementation in the CPFE framework. While the commonly observed $\{10 \overline{1} 2\}$ extension twins are activated in this study, the proposed constitutive laws and implementation can be extended to other twin variants in $h c p$ crystals as well.

\subsection{A Twin Nucleation Model}

A twin nucleation model, along with its numerical implementation in CPFE framework, has been described in (Cheng and Ghosh, 2015). Twin nucleation occurs by a nonplanar dissociation process of a sessile pyramidal $\langle c+a\rangle$ dislocation. The sessile pyramidal $\langle c+a\rangle$ dislocation dissociates into a multi-layer twin nucleus, which propagates 
Table 1: Crystal plasticity constitutive parameters for the dislocation slips in pure Mg.

\begin{tabular}{|c|c|c|c|c|c|}
\hline$C_{11}(\mathrm{GPa})$ & $C_{12}(\mathrm{GPa})$ & $C_{13}(\mathrm{GPa})$ & $C_{33}(\mathrm{GPa})$ & $C_{44}(\mathrm{GPa})$ & \\
\hline 59.40 & 25.61 & 21.44 & 61.6 & 16.4 & \\
\hline$m$ & $\dot{\gamma}_{0}\left(s^{-1}\right)$ & $r$ & $Q_{\text {slip }}(\mathbf{J})$ & & \\
\hline 0.02 & 0.0023 & 0.9 & $1.6 \times 10^{-19}$ & & \\
\hline$\left(s_{*, 0}^{\alpha}\right)_{\text {bas }}(\mathrm{MPa})$ & $\left(s_{*, 0}^{\alpha}\right)_{\text {pri }}(\mathrm{MPa})$ & $\left(\bar{c}_{*, 0}^{\alpha}\right)_{\text {pyr }}(\mathrm{MPa})$ & & & \\
\hline 1.4 & 32.5 & 50 & & & \\
\hline$\left(s_{*, \text { sat }}^{\alpha}\right)_{\text {bas }}(\mathrm{MPa})$ & $\left(s_{*, s a t}^{\alpha}\right)_{p r i}(\mathrm{MPa})$ & $\left(s_{*, s a t}^{\alpha}\right)_{p y r}(\mathrm{MPa})$ & & & \\
\hline 30.0 & 85.0 & 155.0 & & & \\
\hline$\left(s_{a, 0}^{\alpha}\right)_{b a s}(\mathrm{MPa})$ & $\left(s_{a, s a t}^{\alpha}\right)_{b a s}(\mathrm{MPa})$ & $\left(s_{a, s a t}^{\alpha}\right)_{p r i}(\mathrm{MPa})$ & $\left(s_{a, s a t}^{\alpha}\right)_{p y r}(\mathrm{MPa})$ & & \\
\hline 0.0 & 20.0 & 45.0 & 85.0 & & \\
\hline$\left(h_{*, r e f}^{\alpha}\right)_{b a s}(\mathrm{MPa})$ & $\left(h_{*, r e f}^{\alpha}\right)_{p r i}(\mathrm{MPa})$ & $\left(h_{*, r e f}^{\alpha}\right)_{\text {pyr }}(\mathrm{MPa})$ & $c_{1}$ & $c_{2}$ & $c_{3}$ \\
\hline 10 & 800 & 1650 & 0.1 & 2.0 & 1.0 \\
\hline$\left(h_{a, r e f}^{\alpha}\right)_{b a s}(\mathrm{MPa})$ & $\left(h_{a, r e f}^{\alpha}\right)_{p r i}(\mathrm{MPa})$ & $\left(h_{a, r e f}^{\alpha}\right)_{p y r}(\mathrm{MPa})$ & $\chi_{\alpha \beta}$ & & \\
\hline 10 & 450 & 550 & 1.0 & & \\
\hline
\end{tabular}

under an applied resolved shear stress and leaves behind a residual stair-rod dislocation to conserve the Burgers vector. The elastic theory of dislocations (Hirth and Lothe, 1982) is adopted to analyze the change of energy required for the dissociation process. The system initial energy $E_{i n i}$ prior to dissociation, defined as the dislocation line self-energy of the sessile pyramidal $\langle c+a\rangle$ dislocation, is expressed as:

$$
E_{i n i}=\frac{L}{4 \pi}\left[K_{i n i}^{e}\left(b_{i n i}^{e}\right)^{2}+K_{i n i}^{s}\left(b_{i n i}^{s}\right)^{2}\right] \ln \frac{R}{r_{0}}
$$

where $K_{\text {ini }}$ is the dislocation elastic energy prefactor that is obtained by an integral method in (Hirth and Lothe, 1982). The superscripts $e$ and $s$ refer to variables related to edge and screw dislocations respectively. The scalar $b$ denotes the magnitude of the dislocation Burgers vector and $R$ and $r_{0}$ are the outer and inner radius of the dislocation core respectively. $L$ is the spatially varying length of the sessile $\langle c+a\rangle$ dislocation. After the dissociation commences, the total system energy $E_{F}$ includes the self-energy of the twinning dislocation loop $E_{t w}$, the self-energy of the stair-rod dislocation $E_{r}$, the twinning stacking fault energy $E_{\text {fault }}$, the interaction energy $E_{\text {int }}$ and the applied external work $W_{\text {ext }}$. They are respectively expressed as:

$$
\begin{aligned}
& E_{F}=E_{t w}+E_{r}+E_{\text {int }}+E_{\text {fault }}-W_{\text {ext }} \\
& E_{t w}=\frac{1}{4 \pi}\left\{L\left[K_{t w}^{s, f t}\left(b_{t w}^{s, f t}\right)^{2}+K_{t w}^{e, f t}\left(b_{t w}^{e, f t}\right)^{2}\right]+2 d\left[K_{t w}^{s, t r}\left(b_{t w}^{s, t r}\right)^{2}\right.\right.
\end{aligned}
$$




$$
\begin{aligned}
& \left.\left.+K_{t w}^{e, t r}\left(b_{t w}^{e, t r}\right)^{2}\right]\right\} \ln \frac{R}{r_{0}}-d\left\{K _ { t w } ^ { e , t r } \left[\frac{\left(\mathbf{b}_{t w}^{e, t r} \otimes \boldsymbol{\xi}^{t r}\right) \cdot\left(\mathbf{b}_{t w}^{e, t r} \otimes \boldsymbol{\xi}^{t r}\right)}{2 \pi} \ln \frac{L}{r_{0}}\right.\right. \\
& \left.\left.+\frac{\left[\left(\mathbf{b}_{t w}^{e, t r} \otimes \boldsymbol{\xi}^{t r}\right) \cdot \mathbf{L}\right]\left[\left(\mathbf{b}_{t w}^{e, t r} \otimes \boldsymbol{\xi}^{t r}\right) \cdot \mathbf{L}\right]}{2 \pi L^{2}}\right]\right\}-d\left\{K_{t w}^{s, t r} \frac{\left(b_{t w}^{s, t r}\right)^{2}}{2 \pi} \ln \frac{L}{r_{0}}\right\} \\
E_{r}= & \frac{L}{4 \pi}\left[K_{r}^{e}\left(b_{r}^{e}\right)^{2}+K_{r}^{s}\left(b_{r}^{s}\right)^{2}\right] \ln \frac{R}{r_{0}} \\
E_{\text {int }}= & -L K_{t w}^{s, f t} \frac{\left(\mathbf{b}_{t w}^{s, f t} \cdot \boldsymbol{\xi}^{f t}\right)\left(\mathbf{b}_{r}^{s} \cdot \boldsymbol{\xi}^{r}\right)}{2 \pi} \ln \frac{d}{r_{0}}-L K_{t w}^{e, f t}\left\{\frac{\left(\mathbf{b}_{t w}^{e, f t} \otimes \boldsymbol{\xi}^{f t}\right) \cdot\left(\mathbf{b}_{r}^{e} \otimes \boldsymbol{\xi}^{r}\right)}{2 \pi} \ln \frac{d}{r_{0}}\right. \\
& \left.+\frac{\left[\left(\mathbf{b}_{t w}^{e, f t} \otimes \boldsymbol{\xi}^{f t}\right) \cdot \mathbf{d}\right]\left[\left(\mathbf{b}_{r}^{e} \otimes \boldsymbol{\xi}^{r}\right) \cdot \mathbf{d}\right]}{2 \pi d^{2}}\right\} \\
E_{\text {fault }}= & \nu_{t w} d L
\end{aligned}
$$

$K_{t w}$ and $K_{r}$ are the elastic energy prefactors for twin and stair-rod partial dislocations respectively. Superscripts $f t$ and $t r$ refer to the front and transverse segments of the partial dislocation loop. The twin and stair-rod partial dislocation are assumed to have the same outer and inner core radius $R$ and $r_{0}$ as the initial sessile $\langle c+a\rangle$ dislocation. The front segment of the twin partial dislocation is assumed to have the same length $L$ and direction as the initial $\langle c+a\rangle$ dislocation. $\mathbf{L}$ is the vector representation of $L$. The length of the transverse segment of the twinning dislocation is taken as the dissociation distance $d . \boldsymbol{\xi}^{f t}$ and $\boldsymbol{\xi}^{t r}$ are the unit vectors of the front and transverse segments of the twin partial dislocation respectively, $\boldsymbol{\xi}^{r}$ is the unit vector of the stair-rod dislocation and $\nu_{t w}$ is the $\{10 \overline{1} 2\}$ twin boundary energy. $\mathbf{b}_{t w}$ and $\mathbf{b}_{r}$ are the Burgers vector of the twin and stair-rod partial dislocations respectively, with $b_{t w}$ and $b_{r}$ denoting their magnitudes. $\tau_{t w}$ is the applied resolved shear stress on the twin dislocation loop. Critical parameters in the twin nucleation model in equation (16) have been calibrated from experimental results in (Cheng and Ghosh, 2015).

The analysis suggests that a stable twin will nucleate if the following energy-based criteria are satisfied:

- Dissociation condition $E_{i n i} \geq E_{t w}(d=0)+E_{r}$, such that the two partial dislocations are energetically favorable for spontaneous dissociation before they separate. This rule reduces to the classic Franks rule for dislocation dissociation $b_{i n i}^{2} \geq b_{t w}^{2}+b_{r}^{2}$ under the condition of isotropy.

- Irreversibility condition $E_{i n i}>E_{F}\left(d=d_{s}, \tau_{t w}\right)$. After dissociation, the two partial 
dislocations separate from each other with the driving force derived from the interaction energy $E_{\text {int }}$, stacking fault energy $E_{\text {fault }}$ and applied external work $W_{\text {ext }}$. The energy at a stable separation distance must be smaller than the initial energy $E_{i n i}$ such that the equilibrium state is energetically favorable and the process is irreversible. The stable separation distance $d_{s}$ corresponds to the minimum of the total post-dissociation energy $E_{F}$ and is obtained as:

$$
\frac{\partial E_{F}}{\partial d}=0, \text { and } \frac{\partial^{2} E_{F}}{\partial d^{2}} \geq 0
$$

- Reliability condition $d_{s}>2 r_{0}$. Below the minimum separation distance $2 r_{0}$ the elastic calculation of the dislocation self-energies are not reliable. Also, below this threshold distance the cores of the initial dislocation, stair rod, and twinning partial dislocations are not distinguishable.

Upon the satisfaction of the above three criteria, it is considered that dissociation has successfully created twin partials and faulted areas, and hence a twin nucleus is formed. To numerically implement into a CPFE framework, the proposed twin nucleation criteria requires upscaling from the length scale of dislocation dissociation, to the length scale of finite elements (integration point). This is discussed in section 3.3. The twin nucleation model parameters for pure $\mathrm{Mg}$ have been determined in (Cheng and Ghosh, 2015) and are listed in table 2.

Table 2: Constitutive parameters for the twin nucleation in pure $\mathrm{Mg}$.

\begin{tabular}{|c|c|c|c|c|c|}
\hline$K_{t w}^{e, f t} \mathrm{~mJ} / \mathrm{mm}^{2}$ & $K_{t w}^{s, f t} \mathrm{~mJ} / \mathrm{mm}^{2}$ & $K_{t w}^{e, t r} \mathrm{~mJ} / \mathrm{mm}^{2}$ & $K_{t w}^{s, t r} \mathrm{~mJ} / \mathrm{mm}^{2}$ & $K_{i n i}^{e} \mathrm{~mJ} / \mathrm{mm}^{2}$ & $K_{i n i}^{s} \mathrm{~mJ} / \mathrm{mm}^{2}$ \\
\hline 24.7 & 17.3 & 24.7 & 17.3 & 24.2 & 17.7 \\
\hline \hline$K_{r}^{e} \mathrm{~mJ} / \mathrm{mm}^{2}$ & $K_{r}^{s} \mathrm{~mJ} / \mathrm{mm}^{2}$ & $\mathrm{n}$ & $L_{0}(n m)$ & $R(n m)$ & $r_{0}(n m)$ \\
\hline 24.5 & 17.3 & 8 & 80 & 1000 & 0.0396 \\
\hline
\end{tabular}

\subsection{The Proposed Twin Propagation Model}

After nucleation of a stable micro-twin, it glides rapidly on twin planes and forms a twin band. Boundaries of the twin band migrate from one twin plane to adjacent twin planes, thus causing an increase in the twin band thickness (Wang and Hirth, 2009). The motion of twins, gliding on twin planes, occurs by a mixed shear-shuffle process (Serra et al., 1991). The shear process causes plastic flow similar to dislocation slip and is characterized by a Burgers vector $b_{t w}$ on a twin system. After unidirectional shearing, the twin partial dislocations cannot progress due to high energy barriers. Such a configuration is known as the pseudo-twin configuration. Corrective non-unidirectional atomic shuffling 
or re-ordering is subsequently required to place atoms into low-energy symmetric positions, which then allows the twin partial dislocations to glide further. A thermal activation based model has been derived in (Keshavarz and Ghosh, 2013) to account for the atomic shear-shuffle process. Assuming that the shear and shuffle processes are both irreversible, the velocity of twin partial dislocation gliding on a twin plane is expressed as:

$$
v_{\text {glide }}=f_{\text {shuffle }} \lambda_{\text {shear }}\left[\exp \left(-\frac{\triangle F-\tau A_{P} b_{t w}}{K_{B} T}\right)\right]
$$

Here $f_{\text {shuffle }}$ is the frequency of shuffling, $\lambda_{\text {shear }}$ is the shear distance and the term $\exp \left(-\frac{\triangle F-\tau A_{P} b_{t w}}{K_{B} T}\right)$ is the probability of gliding in the presence of an internal energy barrier $\triangle F . \tau$ is the effective resolved shear stress on the twin plane and $A_{p}$ is the shearing area during the plastic deformation.

The twin boundary migrates when the twin partial dislocation glides on the adjacent twin plane. The growth velocity, perpendicular to the twin plane, can be obtained from a stimulated slip model developed in (Yu et al., 2010). This model assumes that dislocation poles promote the transfer of twin-partial dislocations from the current twin plane to adjacent twin planes. Let $\rho_{\text {tot }}$ be the total dislocation density and $P_{\text {promoter }}$ be a small fraction of dislocations that penetrate the twin planes and act as promoters. For moving the twin partial dislocations with a velocity $v_{\text {glide }}$ and by a length $l_{t w}$, the rate of encountering a promoter is given as:

$$
R=P_{\text {promoter }} \rho_{\text {tot }} v_{\text {glide }} l_{t w}
$$

The average time required to meet a promoter is inversely proportional to the rate as $\triangle t_{t w}=\frac{1}{R}$. If $d_{t w}$ denotes the distance between twin planes, then the velocity of twin partial dislocations crossing twin planes is given as the distance divided by the time interval:

$$
v_{\text {grow }}=\frac{d_{t w}}{\triangle t_{t w}}=d_{t w} P_{\text {promoter }} \rho_{\text {tot }} l_{\text {tw }} v_{\text {glide }}
$$

For the stimulated slip model, the twin continuously glides on adjacent planes. Correspondingly the time-averaged plastic shear-rate due to micro-twinning can be obtained from the Orowan equation, written in terms of the gliding velocity $v_{\text {glide }}$, the density of twin partial dislocation $\rho_{t w}$ and its Burgers vector as:

$$
\dot{\gamma}_{t w}=\rho_{t w} b_{t w} v_{\text {glide }}
$$

Substituting equation (18) into the Orowan equation, the twin system shear rate in equation (4) is obtained as:

$$
\dot{\gamma}_{t w}=\dot{\gamma}_{0, t w} \exp \left(-\frac{\triangle F-\tau A_{P} b_{t w}}{K_{B} T}\right)
$$


where $\dot{\gamma}_{0, t w}=\rho_{t w} b_{t w} f_{\text {shuffle }} \lambda_{\text {shear }}$.

While there are many similarities in the way deformation twinning causes shear with pure dislocation slip, there are some differences that should be accounted for in the constitutive relation. Both slip and deformation twinning progresses by shear on the crystallographic lattice. In pure slip, the lattice configuration is not altered and many dislocations can glide on the same lattice plane. The shear associated with slip may be many times the Burgers vector $b$, depending on the number of dislocations involved, as illustrated in figure $2 \mathrm{a}$. On the other hand, deformation twinning reorients the lattice into a mirror symmetric configuration and no subsequent twin can glide on a twinned lattice plane. The shear associated with deformation twinning is uniformly distributed over a volume as illustrated in figure $2 \mathrm{~b}$. The maximum slip $\gamma_{t w}^{\max }$ that occurs by deformation twinning in a given volume varies with the twin type. For example, for $\{10 \overline{1} 2\}$ twin in $\mathrm{Mg}, \gamma_{t w}^{\max }=0.1289$. In addition, the shearing associated with slip can be positive or negative, depending on the direction of dislocation slip and the local stress. Deformation twinning can only provide shear in one direction, which leads to the mirror symmetric lattice configuration. Thus, shear associated with deformation twinning should satisfy the constraint $0 \leq \gamma_{t w} \leq \gamma_{t w}^{\max }$.

The shear resistance of twin partial dislocations changes when it interacts with dislocations, precipitates or other twin variants. The twin system hardening can be caused by its interaction with mobile dislocations, which can impact the twin boundary and create local surface steps (Wang and Agnew, 2015). Alternatively, it can harden by interaction with immobile forest dislocations, precipitates and existing twins that hinder the twin gliding or growth. Though equation (22) does not explicitly contain the shear resistance term and hence cannot directly model hardening of twin systems, it can be reduced to a conventional shear resistance-based power law model using ideas in (Hull and Bacon, 2001). This is derived next.

No thermal activation takes place at a temperature of $0 K$. Any local obstacle with an internal energy barrier $\triangle F$ must be overcome before further twin gliding occurs by the applied stress $\tau(T=0)$, i.e., $\triangle F=\tau(T=0) V^{*}$, where $V^{*}=A_{p} * b_{t w}$ is the activation volume. Substituting this relation into equation (22), the twin shear rate at a temperature $T$ can be written as:

$$
\dot{\gamma}_{t w}=\dot{\gamma}_{0, t w} \exp \left[-\frac{\triangle F}{K_{B} T}\left(1-\frac{\tau(T)}{\tau(T=0)}\right)\right]
$$

For moderate rates of plastic deformation, the temperature dependent shear stress factor $\frac{\tau(T)}{\tau(0)}$ is of the order $\mathrm{O}(1)$. Thus the exponential term $\exp \left(\frac{\tau(T)}{\tau(0)}-1\right)$ can be expanded in a 
series and only the linear term $\frac{\tau(T)}{\tau(0)}$ retained in the relation. In addition, by using the scalar relation $\exp (b a)=(\exp (a))^{b}$, equation (23) can be rewritten in a power-law form as:

$$
\dot{\gamma}_{t w}=\dot{\gamma}_{0, t w}\left|\frac{\tau(T)}{\tau(T=0)}\right|^{\frac{\Delta F}{K_{B} T}} \operatorname{sign}(\tau(T))
$$

At $0 \mathrm{~K}$ temperature the magnitude of the twin shear resistance $s_{t w}$ is equal to the resolved shear stress $\tau(T=0)$. Replacing $\tau(T=0)$ in equation (24) with $s_{t w}$, the conventional power-law plastic slip rate model is obtained for deformation twinning. A phenomenological hardening law is adopted for $s_{t w}$ due to interactions with dislocation slip. For a twin system $\alpha$, the rate of shear resistance is expressed as:

$$
\dot{s}_{t w}^{\alpha}=\sum_{\beta=1}^{N_{\text {slip }}} h^{\alpha \beta}\left|\dot{\tilde{\gamma}}^{\beta}\right|
$$

where the hardening matrix $h^{\alpha \beta}$ is expanded to quantify hardening due to dislocation slip in the twinned regions and is given in equation (8). The hardening of twin systems due to twin-twin interactions are not considered in the this work. The twin boundaries act as barriers to dislocation slips and causes hardening on slip systems. The hardening of slip systems due to twinning is expressed as:

$$
\dot{s}_{\text {slip-twin }}^{\alpha}=\sum_{\beta=1}^{N_{t w i n}} h^{\alpha \beta}\left|\dot{\gamma}_{t w}^{\beta}\right|
$$

\subsection{Numerical Implementation of The Twin Nucleation and Propagation Models}

The micro-twin nucleation and propagation models are explicitly implemented in the CPFE analysis framework. The implementation scheme is illustrated in figure 3 , where twins evolve in two stages. In stage 1, the twin nucleation criteria for CPFE analyses is developed by upscaling the atomic-length-scale mechanics model in section 3.3.1 to continuum scales. All elements in each grain at each time increment are checked for potential twin nucleation. If an element satisfies the upscaled nucleation criteria, it is identified as a twin nucleation site. Stage 2 starts after a twin has nucleated in any grain. In the subsequent time steps, all elements in the parent grain of the twin are checked for twin band propagation and thickening, using criteria delineated in section 3.3.2.

\subsubsection{Implementation of the Twin Nucleation Model}

The twin nucleation criterion in section 3.1 is based on the dislocation dissociation mechanisms at the length-scale of individual dislocations. A few assumptions are made 


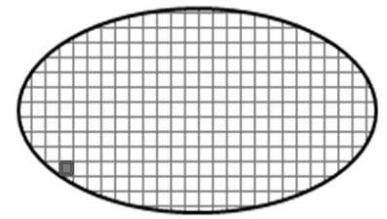

Identify twin nucleation site

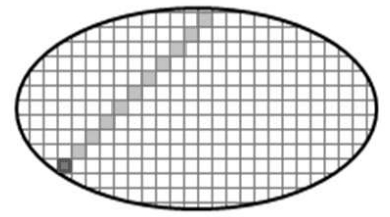

Check neighboring elements for twin propagation

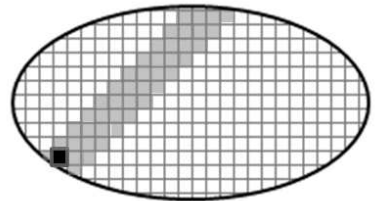

Check neighboring element for twin band thickening

Stage 1

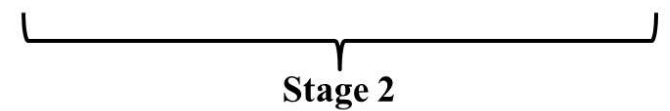

Figure 3: Illustration of stages of twin nucleation and propagation in the CPFE model.

to apply this model to the continuum scale of constitutive models in CPFE models of polycrystalline microstructures, where each element (integration point) is of the order of $\leq 100 \mathrm{~nm}$. Underlying each integration point, multiple sessile $\langle c+a\rangle$ dislocations can exist acting as twin nucleation sources. The length $L$ of sessile $\langle c+a\rangle$ dislocations can vary spatially over the entire microstructural volume modeled. However, a constant value $L=L_{0}$ is used for all sessile $\langle c+a\rangle$ dislocations in the present work. Also, the local 2nd Piola-Kirchhoff stress $\mathbf{S}^{l o c}$ in the vicinity of sessile $\langle c+a\rangle$ dislocations can vary due to stress fields of the distributed nano-scale dislocation debris, point defect density and impurity content. The stress at each integration point $\mathbf{S}^{G P}$ is considered to be the homogenized value of spatially varying $\mathbf{S}^{l o c}$. Similar considerations have also been made for VPSC model in (Beyerlein et al., 2011). In the work, the local stress state $\mathbf{S}^{l o c}$ is identified with its average, i.e. $\mathbf{S}^{l o c}=\mathbf{S}^{G P}$.

The twin nucleation criteria is then adapted for the CPFE modeling as:

$$
\begin{aligned}
& E_{i n i} \geq E_{t w}\left(d=0, L_{0}\right)+E_{r} \text { and } \\
& E_{i n i}>E_{F}\left(d_{s}, L_{0}, \tau_{t w}^{G P}\right) \forall d_{s}>2 r_{0}
\end{aligned}
$$

where $\tau_{t w}^{G P}$ is the resolved shear stress on a twin system projected from the stress $\mathbf{S}^{G P}$ at an integration point. A total of six $\{10 \overline{1} 2\}$ twin variants are considered at each integration point. For a twin variant $\alpha$, the resolved shear stress $\tau_{t w}^{\alpha}$ is expressed as $\tau_{t w}^{\alpha}=$ $\left(\mathbf{F}^{e T} \mathbf{F}^{e} \mathbf{S}^{G P}\right):\left(\mathbf{s}_{\mathrm{tw}, \mathbf{0}}^{\alpha}\right)$. When one twin variant first satisfies the nucleation criteria (27), this integration point is considered as a twin nucleus, from which the twin starts to propagate. 


\subsubsection{Numerical Implementation of Twin Propagation Model}

For a twin partial dislocation to glide to a neighboring point $\mathrm{X}$ in the nucleation plane, the propagation velocity in the plane must exceed a critical value. This is expressed as:

$$
v_{\text {glide }} \geq \frac{l_{\text {glide }}}{\triangle t_{\text {twin }}}
$$

where $l_{\text {glide }}$ is the distance between the neighboring point $\mathrm{X}$ and the nucleation site, and $\triangle t_{\text {twin }}$ is the time interval from the time of twin nucleation $t_{\text {nucleation }}$ to the current time $t$. Substituting equation (18) into equation (28), the criterion for a micro-twin to propagate to a neighboring point $\mathrm{X}$ is established as:

$$
\tau_{\text {crit }} \geq \frac{\ln \left(\frac{l_{\text {growth }}}{\Delta t_{\text {twin }} f_{\text {shuffle }} \lambda_{\text {shear }}}\right) K_{B} T+\triangle F}{A_{p} b_{t w}}
$$

Thickening by growth of twins is implemented by considering a twin partial dislocation to move transversely to a neighboring point $X$ that is located outside of the twin nucleation plane. The twin needs to move in two directions, viz. normal to the twin plane with a velocity $v_{\text {grow }}$ and along the twin plane with a velocity $v_{\text {glide }}$ to propagate to this neighboring point. This is illustrated in figure 4 . Additional conditions over that in equation (28) are needed to achieve this state. If $l_{\text {growth }}$ is the normal distance from the point $\mathrm{X}$ to the twin plane, the additional requirement for twin velocity is:

$$
v_{\text {grow }} \geq \frac{l_{\text {growth }}}{\triangle t_{\text {twin }}}
$$

while the additional critical stress requirement for twin propagation is given as:

$$
\tau_{\text {crit }} \geq \frac{\ln \left(\frac{l_{\text {glide }}}{\Delta t_{\text {twin }} f_{\text {shuffle }} \lambda_{\text {shear }} d_{t w} P_{\text {promoter }} \text { tot }_{\text {t }} l_{\text {tw }}}\right) K_{B} T+\triangle F}{A_{p} b_{t w}}
$$

Equations (29) and (31) provide quantitative measures to propagate a twin to a neighboring material point in longitudinal and transverse (through-thickness) directions respectively. In the CPFE implementation with incremental time-marching process, this is executed explicitly at the end of each time increment.

The non-local twin propagation model has an intrinsic size-dependence due to the growth-propagation relations. This is illustrated through a numerical example for two single crystals. The single crystals are respectively of size $20 \mu m \times 10 \mu m \times 10 \mu m$ (L) and $10 \mu m \times 5 \mu m \times 5 \mu m(S)$, and are subjected to a uniaxial constant applied strain-rate loading of $10^{-3} / \mathrm{s}$. A twin of initial thickness $1 \mu \mathrm{m}$ is seeded in each of the two samples, 


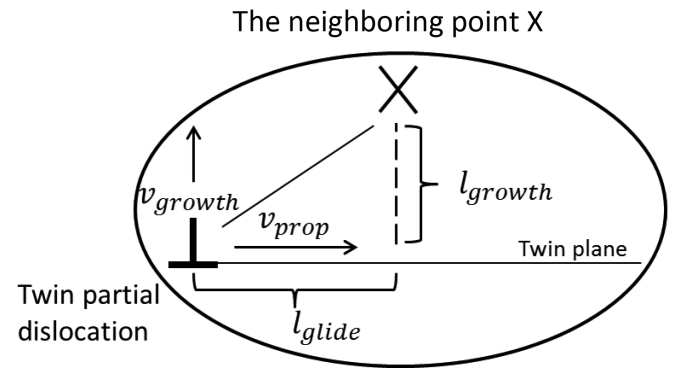

Figure 4: Illustration of a twin partial dislocation propagation and growth (thickening) to a neighboring point $\mathrm{X}$ by respectively gliding on the twin plane and growing normal to it.

as shown in figures 5a and 5b. With increasing load, the twin will thicken and induce shear deformation. Nucleation of new twins is turned off in these simulations and only twin growth is tested. Figure 5c plots the volume fraction of twinned elements in the computational domain as a function of the overall strain. The twin thickening speed is nearly the same for the two tests, which causes the twin volume fraction in the smaller crystal (S) to increase at a faster rate. This results in a higher slip system hardening rate in the smaller crystal (S) due to a higher volume fraction of twins.

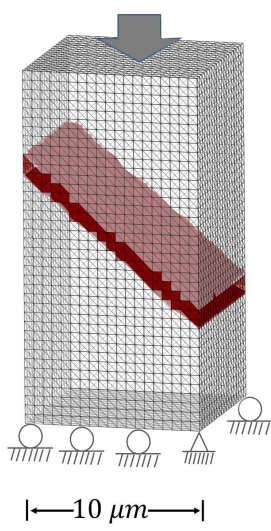

(a)

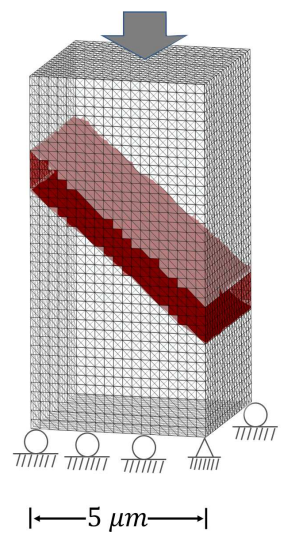

(b)

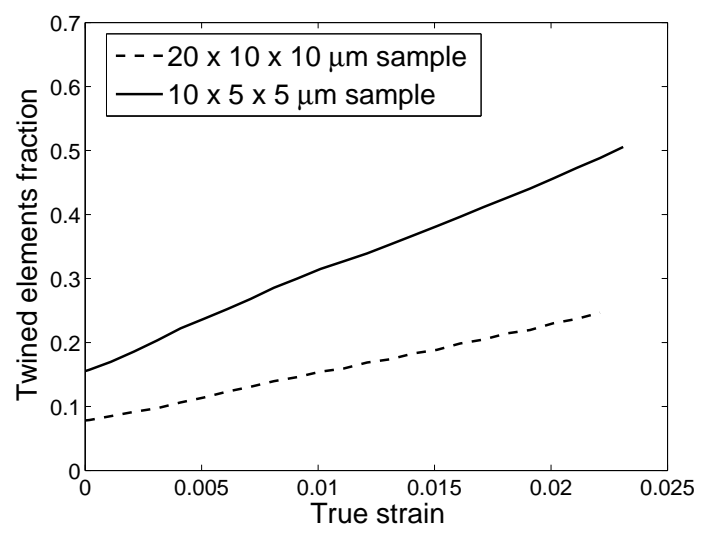

(c)

Figure 5: Simulation results for twin growth in two single crystals with identical initial twins: the crystals with growing twins in (a) larger crystal (L) and (b) smaller crystal (S); and (c) the rate of twin volumefraction increase in the model. 


\section{Time Integration Algorithm in Numerical Implementation}

Numerical time integration of the constitutive model in section 2 requires the evaluation of non-local variables related to GND and twins from neighboring elements. The time integration algorithm integrates the set of coupled differential equations in the non-local constitutive model. Integration at each integration (Gauss quadrature) point of an element proceeds in the following three steps:

- Step A: Update stresses, plastic strains and all state variables, keeping the non-local

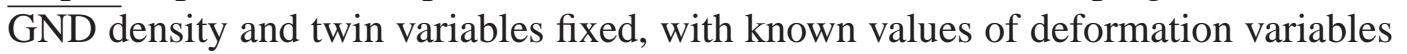
at time $t$, as well as a given deformation gradient $\mathbf{F}(t+\Delta t)$ at time $t+\triangle t$;

- Step B: Update the GND densities and their rates of hardening by evaluating $\nabla_{X} \times$ $\overline{\mathbf{F}^{p T}}$, using values in adjacent elements;

- Step C: Check all available integration points for new twin nucleation and propagate the existing twins.

Note that step A is performed locally at each Gauss quadrature point, whereas steps B and $\mathrm{C}$ require obtaining variables from neighboring elements. An implicit update algorithm, proposed in (Balasubramanian, 1998) and extended in (Cheng and Ghosh, 2015), is implemented in step A with updates in steps B and C. For step A, the algorithm assumes that the primary unknown variable is the second Piola-Kirchhoff stress $\mathbf{S}$, and seeks its solution from a set of six nonlinear equations by a Newton-Raphson type iterative solver. Other deformation and state variables are updated during the iterations. The six nonlinear equations correspond to the six components of $\mathbf{S}$ that are given as (Cheng and Ghosh, 2015):

$$
\mathbf{S}(t+\triangle t)=\mathbf{S}^{t r}-\sum_{\alpha=1}^{N_{t o t}} \triangle \gamma^{\alpha}\left(\mathbf{S}(t+\triangle t), s_{a}^{\alpha}(t+\triangle t), s_{*}^{\alpha}(t+\triangle t)\right) \mathbf{B}^{\alpha}
$$

where $N_{\text {tot }}$ is the total number of slip systems and twin systems and $\mathbf{B}^{\alpha}$ is defined as:

$$
\begin{array}{r}
\mathbf{B}^{\alpha}=\mathbf{C}:\left[\frac{1}{2}\left(\mathbf{A}(t+\triangle t) \mathbf{s}_{0}^{\alpha}+\mathbf{s}_{0}^{\alpha} \mathbf{A}(t+\triangle t)\right)\right] \text { where } \\
\mathbf{A}(t+\triangle t)=\mathbf{F}^{p^{-T}}(t) \mathbf{F}^{T}(t+\triangle t) \mathbf{F}(t+\triangle t) \mathbf{F}^{p^{-1}}(t)
\end{array}
$$

$\mathbf{S}^{t r}$ is the trial stress, expressed as:

$$
\mathbf{S}^{t r}=\mathbf{C}: \frac{1}{2}(\mathbf{A}(t+\triangle t)-\mathbf{I})
$$


The nonlinear equation (32) is solved using the Newton-Raphson iterative solver. Defining a residual as:

$$
\mathbf{G}\left(\mathbf{S}^{i}(t+\triangle t)\right)=\mathbf{S}^{i}(t+\triangle t)-\mathbf{S}^{t r}+\sum_{\alpha=1} \triangle \gamma^{\alpha} \mathbf{B}^{\alpha}
$$

the $i-t h$ iteration update to $\mathbf{S}$ is obtained as:

$$
\mathbf{S}^{i+1}(t+\Delta t)=\mathbf{S}^{i}(t+\Delta t)-\left.\frac{\partial \mathbf{G}}{\partial \mathbf{S}}\right|_{i} ^{-1} \mathbf{G}\left(\mathbf{S}^{i}(t+\Delta t)\right)
$$

In this update procedure for $\mathbf{S}(t+\triangle t)$, the slip system resistances are held fixed. After convergence, the increment of shear resistances from SSDs and activated twin system hardening variables are updated using equations (7a), (7b) and (25). The GNDs are kept fixed at their values at time $t$ in this step.

The next step B computes the GND densities and associated hardening increments. A non-local algorithm is implemented to evaluate the Nyes dislocation density tensor $\Lambda$ involving the gradient operator. The local gradient operator in an element is calculated from nodal values of the variable using element shape functions. The super-convergent patch recovery (SPR) method (Zienkiewicz and Zhu, 1992) is implemented to evaluate the nodal values of $\mathbf{F}_{p}$ from Gauss quadrature points to evaluate $\nabla_{X} \times \mathbf{F}^{p T}$ at quadrature points (Cheng and Ghosh, 2015).

Step $\mathrm{C}$ updates the twin domain by nucleating new twins as well as by propagating and growing already nucleated twins. A straight-forward scheme to update twins is by using an explicit staggered update algorithm, where twin domains are updated at the end of each time-step explicitly. However, this staggered algorithm inaccurately predicts twin configurations and deformation when the time-step exceeds a critical value for cases when the rate of twin nucleation and evolution is higher than the rate of deformation. This is discussed in details in section 5.4. An implicit subcycling algorithm is proposed in section 5 to overcome the time-step constraint. Steps needed for numerically integrating the nonlocal crystal plasticity constitutive models are sequentially summarized in the flow chart 3 .

\subsection{Numerical Instabilities of CPFE Model with Twinning}

The CPFE model with twinning can suffer from numerical instabilities, even for quasistatic processes, when time-steps are large. The evolving twins pose severe constraints on time-steps, due to high propagation-rate and localized plastic flow in the twinned regions. The instability should be resolved prior to discrete twin prediction. 
Table 3: Flowchart showing computational operations in the constitutive update procedure.

Step A Update local stress and deformation variables from $t$ to $t+\Delta t$, with known $\mathbf{F}(t+\triangle t)$ and other variables at time $t$

i Calculate trial second Piola-Kirchhoff stress $\mathbf{S}^{\text {tr }}$ from equation (34), and update slip rates $\triangle \gamma^{\alpha}$ using equations (6).

ii Obtain the starting value $\mathbf{S}^{1}(t+\triangle t)$ from equation (32)

iii For the $i$-th iteration step in the Newton-Raphson algorithm:

(a) Compute residual $\mathbf{G}$ from equations (35)

(a) Update $\mathbf{S}^{i+1}(t+\triangle t)$ from equations (36)

(c) Check for convergence: if no, go back to sub-step $(a)$; if yes, proceed to step iv.

iv Update slip system shear resistance with SSDs hardening using equations (7) and (8)

v Check for convergence of SSDs: if no, go back to step iii and compute stress with updated slip system shear resistance; if yes, proceed to step vi

vi Evaluate $\mathbf{F}^{p}(t+\triangle t)$. Also calculate $\mathbf{F}^{e}(t+\triangle t)=\mathbf{F}(t+\triangle t) \mathbf{F}^{p^{-1}}(t+\triangle t)$, the Cauchy stress $\sigma(t+\triangle t)=\frac{1}{\operatorname{det}\left(\mathbf{F}^{e}(t+\triangle t)\right)} \mathbf{F}^{e T}(t+\triangle t) \mathbf{S}(t+\triangle t) \mathbf{F}^{e}(t+$ $\triangle t)$ and the elasto-plastic tangent stiffness matrix $\mathbb{C}=\frac{\partial \sigma}{\partial \varepsilon}$.

vii Execute step B

Step B Update non-local deformation variables related to GNDs

i Compute the nodal values of $\mathbf{F}^{p}(t+\triangle t)$ with SPR method and obtain $\nabla_{X} \times \mathbf{F}^{p^{T}}$ (Cheng and Ghosh, 2015)

ii Compute the Nye's tensor $\Lambda$ and obtain GND density from equation (11)

iii Update slip system shear resistance with GND hardening from equations (9) and (7).

iv Execute step $\mathbf{C}$

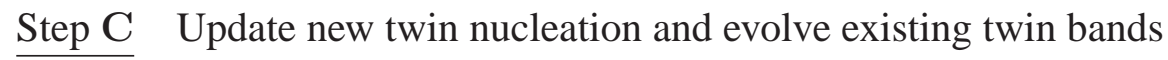

i Identify new twin nucleation sites 2 by checking all elements that have not nucleated or propagated a micro-twin with the nucleation criteria in equation (27).

ii Evolve the existing twin bands by checking all elements in the vicinity of the twin nucleation site with the twin propagation criteria (29) and growth criteria (31). 
The power-law type crystal plasticity constitutive models are reported to be quite stiff (Zhang et al., 2014; Ling et al., 2005; Kuchnick et al., 2006; Roters et al., 2010a). The low strain-rate sensitivity of many metals at room temperature results in a very small value of the rate-sensitivity parameter $m$ in the flow rule equation (6). For example, $m \approx 0.02$ for $\mathrm{Mg}$ at room temperature. Small variations in the resolved shear stress causes large changes in the slip-rates. In equation (6), the slip-rate $\dot{\gamma}$ quickly reaches very high values for $\tau_{e f f}^{\alpha} / s_{*}^{\alpha}>1$. The slope of this non-linear equation also rapidly increases for $\tau_{e f f}^{\alpha}>s_{*}^{\alpha}$. This rapid slip-rate increase causes major convergence issues with the Newton-Raphson solver, according to the condition

$$
\dot{\gamma}^{\alpha} \triangle t \geq \triangle \gamma_{c r i t}
$$

where $\triangle \gamma_{\text {crit }}$ is a critical slip increment (Van der Giessen and Neale, 1993). An alternative criteria is proposed in (Balasubramanian, 1998) as:

$$
\tau_{e f f}^{\alpha} / s_{a}^{\alpha} \leq r_{c r i t}
$$

The ratio $r_{c r i t}=2.0$ in (Balasubramanian, 1998). These criteria establish a critical time step $\triangle t_{\text {crit }}$ for numerical time integration of the crystal plasticity model. Since the slip rate $\triangle \gamma_{c r i t}$ and the resolved shear stress $\tau_{\text {eff }}^{\alpha}$ are implicitly dependent on the time step $\triangle t$ in a backward Euler update algorithm, $\triangle t_{\text {crit }}$ is a function of $\left(m, \gamma_{0}, \mathbf{C}^{e}, s_{a}^{\alpha}, s_{*}^{\alpha}, \tau^{\alpha}, \triangle \gamma_{\text {crit }}, r_{\text {crit }}\right)$. Thus the critical time step evolves with the evolution of internal state variables.

Several numerical methods have been proposed to alleviate this critical time step constraint, e.g., by choosing appropriate starting values for the Newton-Raphson iteration in equation (32) (Roters et al., 2010a), or by adding line search techniques (Ling et al., 2005). While they partially relieve the critical time-step issue and accelerate CPFE analysis, they cannot completely remove the time-step constraints.

Deformation twinning is represented by a power-law model for shearing on twin systems in equation (24). This model imposes an even more stringent instability criterion, since twinning has been reported to be even less strain-rate sensitive, e.g. in (Majkut, 2013). Furthermore, lattice misorientations across grain and twin boundaries renders the deformation to be heterogeneous. Strain localizes inside twin bands, while stress concentrates at the boundaries of grain-twin, twin-twin and grain-grain regions. As a consequence, the distribution of critical time steps inside the microstructural volume is not uniform. Figure 6a shows the strain-rate distributions at $2 \%$ strain in a AZ31 alloy polycrystalline microstructural representative volume element (RVE) containing 152 grains. The results show that the strain-rates inside twin bands is in general $\sim 5-10$ times higher 
than those in matrix. This leads to a heterogeneous distribution of critical time step as plotted in figure 6b. The elements inside twin bands, especially those close to the twin boundaries and grain boundaries, requires much smaller time steps to integrate the twin constitutive equations in comparison with those in outside regions. A quantitative comparison of the critical time step size is tabulated in table 4. It is found $97 \%$ elements can proceed with a time step of $\Delta t=10 s$. However CPFE simulations of the entire RVE has to proceed with a significantly smaller time step of $\Delta t=0.0391 \mathrm{~s}$, required by only $3 \%$ of the elements that are located at the critical regions. This is a reduction in the time step by a factor of $\sim 255$. This is a significant limitation on the efficiency of CPFE simulations using the same time-steps for all elements in the RVE, especially for high resolution large microstructural models.

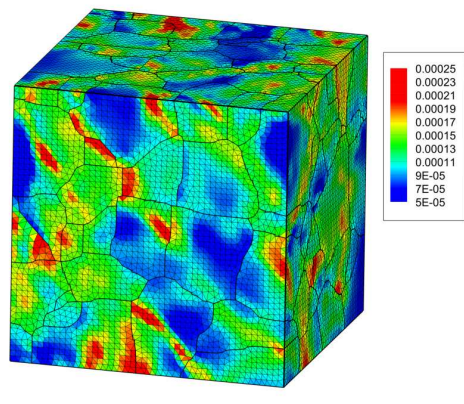

(a)

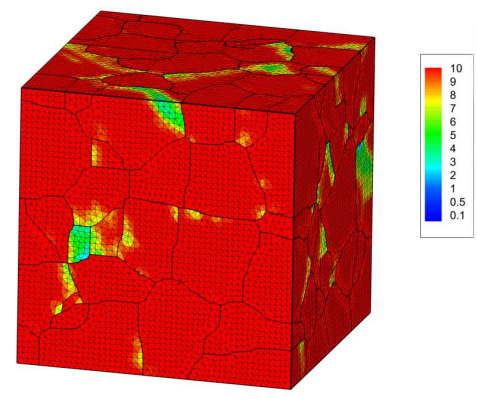

(b)

Figure 6: (a) Strain rate distribution at $2 \%$ strain in a crystal plasticity finite element simulation of AZ31 polycrystal microstructure, (b) distribution of critical time steps for integrating crystal plasticity equations.

\begin{tabular}{|c|c|c|c|c|c|c|c|c|c|}
\hline$\triangle t_{\text {crit }}$ (secs.) & 10 & 5 & 2.5 & 1.25 & 0.625 & 0.313 & 0.156 & 0.0781 & 0.0391 \\
\hline \# of elements & 672868 & 15693 & 7593 & 2171 & 535 & 183 & 61 & 23 & 3 \\
\hline
\end{tabular}

Table 4: Distribution of critical time-step sizes among elements in the ensemble.

\section{Adaptive Subcycling Algorithm for Accelerating CPFE Simulations}

A novel multi-time domain subcycling algorithm is proposed to avert the minimum critical time step constraints, while temporally matching the twin evolution-rate. The proposed subcycling algorithm decomposes the microstructural RVE or statistically equivalent RVE (SERVE) into separate computational domains and simulates each sub-domain 
using a different time step, as required. The simulation is accelerated by adopting a coarse time step for the non-critical regions, while solving regions of localization with fine time steps. Starting with a known state at time $t$, the non-critical sub-domain is solved using a time-increment $\Delta t$, while the localized deformation sub-domain is solved using very fine time steps $\triangle \tau$ from time $t$ to $t+\Delta t$ as shown in figure 7 . A predictor-corrector routine is implemented, where results from each of the domains are assembled for estimating and correcting the error due to this domain decomposition. The interface between the two sub-domains satisfies compatibility through applied displacement boundary conditions, that is a displacement predictor estimated from deformation history. At time $t+\Delta t$, the displacement corrector is obtained by equilibrating the nodal residual forces of the entire microstructural SERVE.

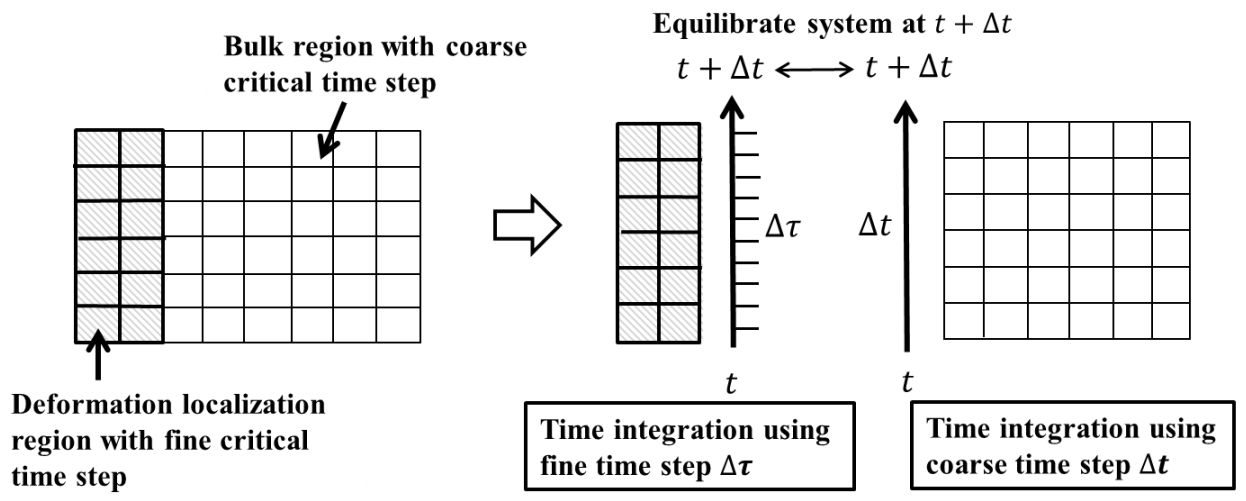

Figure 7: Schematic depiction of the subcycling algorithm.

\subsection{Formulation of Subcycling Algorithm}

In a time increment from time $t$ to $t+\triangle t$, the CPFE analysis solves for the equilibrium state of material undergoing large deformation. The resulting system of non-linear equations can be linearized as (Cheng et al., 2016):

$$
\mathbf{K}_{t} \triangle \mathbf{u}_{t \rightarrow t+\triangle t}=\mathbf{f}_{t+\triangle t}^{e x t}-\mathbf{f}_{t}^{i n t}
$$

where $\mathbf{K}_{t}$ is the global tangent stiffness matrix and $\mathbf{f}_{t+\triangle t}^{e x t}$ and $\mathbf{f}_{t}^{\text {int }}$ are respectively the prescribed applied external force vector at time $t+\Delta t$ and the internal force vector at time $t$, written as:

$$
\mathbf{f}_{t}^{i n t}=\int_{\Omega_{t}} \mathbf{B}^{T} \boldsymbol{\sigma}_{t} d \Omega
$$




$$
\mathbf{f}_{t+\triangle t}^{e x t}=\int_{\Omega_{t+\Delta t}} \mathbf{N}^{T} \mathbf{b}_{t+\triangle t} d \Omega+\int_{\Gamma_{t+\Delta t}^{\overline{\mathbf{t}}}} \mathbf{N}^{T} \overline{\mathbf{t}}_{t+\triangle t} d \Gamma^{\overline{\mathbf{t}}}
$$

Here $\sigma$ is the Cauchy stress, $\mathbf{b}$ is the body force per unit volume, $\overline{\mathbf{t}}$ is the applied traction, $\mathbf{B}$ is the strain-displacement matrix and $\mathbf{N}$ is the shape function matrix. $\Omega$ denotes the volume of the deforming body, $\Gamma^{\overline{\mathrm{t}}}$ is the surface on which traction is applied.

The subcycling algorithm splits the computational domain of the virtual microstructure $\Omega$ into a fine time-scale domain $\Omega^{F}$ and a coarse time-scale domain $\Omega^{C}$. Accordingly, the nodal degrees of freedom $\triangle \mathbf{u}$ can be separated into three parts, viz. $\triangle \mathbf{u}^{F}, \triangle \mathbf{u}^{C}$, and $\triangle \mathbf{u}^{I}$ depending on whether they belong to $\Omega^{F}, \Omega^{C}$ or their interface, $\partial \Omega^{I}$ respectively. The tangent stiffness matrix $\mathbf{K}$ and out-of-balance force vector $\mathbf{f}=\mathbf{f}^{\text {ext }}-\mathbf{f}^{\text {int }}$ can be partitioned as:

$$
\left[\begin{array}{ccc}
\mathbf{K}_{\Omega^{F}}^{F, F} & \mathbf{K}_{\Omega^{F}}^{F, I} & 0 \\
\mathbf{K}_{\Omega^{F}}^{I, F} & \mathbf{K}_{\Omega^{F}}^{I, I}+\mathbf{K}_{\Omega^{C}}^{I, I} & \mathbf{K}_{\Omega^{C}}^{I, C} \\
0 & \mathbf{K}_{\Omega^{C}}^{C, I} & \mathbf{K}_{\Omega^{C}}^{C, C}
\end{array}\right]\left\{\begin{array}{c}
\Delta \mathbf{u}^{F} \\
\Delta \mathbf{u}^{I} \\
\Delta \mathbf{u}^{C}
\end{array}\right\}=\left\{\begin{array}{c}
\mathbf{f}_{\Omega^{F}}^{F} \\
\mathbf{f}_{\Omega^{F}}^{I}+\mathbf{f}_{\Omega^{C}}^{I} \\
\mathbf{f}_{\Omega^{C}}^{C}
\end{array}\right\}
$$

where $\left[\mathbf{K}_{\Omega^{F}}\right]=\left[\begin{array}{ll}\mathbf{K}_{\Omega^{F}}^{F, F} & \mathbf{K}_{\Omega^{F}}^{F, I} \\ \mathbf{K}_{\Omega^{F}}^{I, F} & \mathbf{K}_{\Omega^{F}}^{I, I}\end{array}\right]$ and $\left\{\mathbf{f}_{\Omega^{F}}\right\}=\left\{\begin{array}{c}\mathbf{f}_{\Omega^{F}}^{F} \\ \mathbf{f}_{\Omega^{F}}^{I}\end{array}\right\}$ are the tangent stiffness matrix and the out-of-balance force vector that are integrated and assembled from the fine time-scale elements. Likewise, $\left[\mathbf{K}_{\Omega^{C}}\right]=\left[\begin{array}{cc}\mathbf{K}_{\Omega^{C}}^{I, I} & \mathbf{K}_{\Omega^{C}}^{I, C} \\ \mathbf{K}_{\Omega^{C}}^{C, I} & \mathbf{K}_{\Omega^{C}}^{C, C}\end{array}\right]$ and $\left\{\mathbf{f}_{\Omega^{C}}\right\}=\left\{\begin{array}{l}\mathbf{f}_{\Omega^{C}}^{I} \\ \mathbf{f}_{\Omega^{C}}^{C}\end{array}\right\}$ are the tangent stiffness matrix and the force vector integrated and assembled from the coarse time-scale elements.

The steps in the subcycling algorithm are described below.

1. Compute trial displacement increment $\triangle \mathbf{u}_{t \rightarrow t+\Delta t}^{\text {trial }}$ for the entire domain $\Omega$ using the known global stiffness matrix $\mathbf{K}_{t}$ at time $t$.

2. Use the trial displacement increment at the interface $\left\{\triangle \mathbf{u}_{t \rightarrow t+\Delta t}^{I, t r i a l}\right\}$ as the displacement boundary condition to solve the explicit CPFE problem for only the coarse domain from $t$ to $t+\triangle t$ with a coarse time step increment $\Delta t$. From equation (41), the coarse time-scale domain problem can be solved for the time increment 


$$
\begin{aligned}
t & \rightarrow t+\triangle t \text { by applying the displacement }\left\{\Delta \mathbf{u}_{t \rightarrow t+\Delta t}^{I, t r i a l}\right\} \text { as: } \\
& \quad\left[\mathbf{K}_{\Omega^{C}, t}^{C, C}\right]\left\{\triangle \mathbf{u}_{t \rightarrow t+\triangle t}^{C}\right\}=\left\{\mathbf{f}_{\Omega^{C}, t+\triangle t}^{C, e x t}\right\}-\left\{\mathbf{f}_{\Omega^{C}, t}^{C, \text { int }}\right\}-\left[\mathbf{K}_{\Omega^{C}, t}^{C, I}\right]\left\{\triangle \mathbf{u}_{t \rightarrow t+\Delta t}^{I, t r i a l}\right\}
\end{aligned}
$$

Following the solution of the coarse time-scale domain displacement increment vector $\left\{\triangle \mathbf{u}_{t \rightarrow t+\triangle t}^{C}\right\}$, the corresponding tangent stiffness matrix and force vector can be updated to time $t+\triangle t$ as $\left[\mathbf{K}_{\Omega^{C}, t+\triangle t}\right]$, and $\left\{\mathbf{f}_{\Omega^{C}, t+\triangle t}\right\}$.

3. Use the trial displacement increment at the interface $\left\{\triangle \mathbf{u}_{t \rightarrow t+\Delta t}^{I, t r i a l}\right\}$ as the displacement boundary condition to solve the explicit CPFE problem for only the fine timescale domain from time $t$ to $t+\triangle t$ with sub-divided time increments $\triangle \tau \ll \Delta t$. The displacement boundary condition at the interface for a sub-step $\tau \rightarrow \tau+\Delta \tau$ is linearly interpolated from $\left\{\triangle \mathbf{u}_{t \rightarrow t+\triangle t}^{I, t r i a l}\right\}$ as:

$$
\left\{\mathbf{u}_{\tau+\Delta \tau}^{I, \text { trial }}\right\}=\left\{\mathbf{u}_{\tau}^{I, \text { trial }}\right\}+\frac{\triangle \tau}{\triangle t}\left\{\triangle \mathbf{u}_{t \rightarrow t+\Delta t}^{I, \text { trial }}\right\}
$$

where $\left\{\mathbf{u}_{\tau}^{I, \text { trial }}\right\}$ and $\left\{\mathbf{u}_{\tau+\triangle \tau}^{I, \text { trial }}\right\}$ are the displacements at the interface at times $\tau$ and $\tau+\triangle \tau$, respectively. From equation (41), the fine time-scale domain problem is solved in the increment $\tau \rightarrow \tau+\triangle \tau$ by applying the displacement $\left\{\triangle \mathbf{u}_{\tau \rightarrow \tau+\triangle \tau}^{I, \text { trial }}\right\}$ as:

$$
\left[\mathbf{K}_{\Omega^{F}, \tau}^{F, F}\right]\left\{\triangle \mathbf{u}_{\tau \rightarrow \tau+\triangle \tau}^{F}\right\}=\left\{\mathbf{f}_{\Omega^{F}, \tau+\triangle \tau}^{F, e x t}\right\}-\left\{\mathbf{f}_{\Omega^{F}, \tau}^{F, i n t}\right\}-\left[\mathbf{K}_{\Omega^{F}, \tau}^{F, I}\right]\left\{\triangle \mathbf{u}_{\tau \rightarrow \tau+\triangle \tau}^{I, t r i a l}\right\}
$$

The following variables are obtained at the completion of step 3:

- Fine time-scale domain displacement increments from $t$ to $t+\triangle t:\left\{\triangle \widetilde{\mathbf{u}}_{t \rightarrow t+\Delta t}^{F}\right\}$

- Tangent stiffness matrix of fine time-scale elements at time $t+\triangle t$ : $\left[\widetilde{\mathbf{K}}_{\Omega^{F}, t+\triangle t}\right]$

- Force vector of fine time-scale elements at time $t+\Delta t:\left\{\widetilde{\mathbf{f}}_{\Omega^{F}, t+\triangle t}\right\}$

The symbol ${ }^{\sim}$ indicates that the variables are obtained by solving a sequence of fine time-increment FE problems.

4. Check if the global equilibrium is satisfied, i.e.:

$$
\left\|\left\{\tilde{\mathbf{f}}_{\Omega^{F}, t+\triangle t}^{F}, \tilde{\mathbf{f}}_{\Omega^{F}, t+\triangle t}^{I}+\mathbf{f}_{\Omega^{C}, t+\triangle t}^{I}, \mathbf{f}_{\Omega^{C}, t+\triangle t}^{C}\right\}^{T}\right\| \leq R_{c r i t}
$$

where $R_{\text {crit }}$ is a scalar convergence criterion. 
- if yes, exit iteration. $\left\{\triangle \widetilde{\mathbf{u}}_{t \rightarrow t+\triangle t}^{F}, \triangle \mathbf{u}_{t \rightarrow t+\Delta t}^{I, t r i a l}, \triangle \mathbf{u}_{t \rightarrow t+\Delta t}^{C}\right\}^{T}$ is the solution to equation (39).

- if no, calculate the corrector to the displacement increment vector as:

$$
\begin{aligned}
& \Delta \mathbf{u}_{t \rightarrow t+\Delta t}^{\text {corrector }}=\left\{\begin{array}{c}
\triangle \widetilde{\mathbf{u}}_{t \rightarrow t+\Delta t}^{F} \\
\triangle \mathbf{u}_{t \rightarrow t+\Delta t}^{I, t r i a l} \\
\triangle \mathbf{u}_{t \rightarrow t+\Delta t}^{C}
\end{array}\right\}+\left\{\begin{array}{c}
\triangle \hat{\mathbf{u}}^{F} \\
\triangle \hat{\mathbf{u}}^{I} \\
\triangle \hat{\mathbf{u}}^{C}
\end{array}\right\} \\
& \left\{\begin{array}{c}
\triangle \hat{\mathbf{u}}^{F} \\
\triangle \hat{\mathbf{u}}^{I} \\
\triangle \hat{\mathbf{u}}^{C}
\end{array}\right\}=\left[\begin{array}{ccc}
\widetilde{\mathbf{K}}_{\Omega^{F}}^{F, F} & \widetilde{\mathbf{K}}_{\Omega^{F}}^{F, I} & \mathbf{0} \\
\widetilde{\mathbf{K}}_{\Omega^{F}}^{I, F} & \widetilde{\mathbf{K}}_{\Omega^{F}}^{I, I}+\mathbf{K}_{\Omega^{C}}^{I, I} & \mathbf{K}_{\Omega^{C}}^{I, C} \\
\mathbf{0} & \mathbf{K}_{\Omega^{C}}^{C, I} & \mathbf{K}_{\Omega^{C}}^{C, C}
\end{array}\right]_{t+\Delta t}\left\{\begin{array}{c}
\widetilde{\mathbf{f}}_{\Omega^{F}}^{F} \\
\widetilde{\mathbf{f}}_{\Omega^{F}}^{I}+\mathbf{f}_{\Omega^{C}}^{I} \\
\mathbf{f}_{\Omega^{C}}^{C}
\end{array}\right\}_{t+\Delta t}
\end{aligned}
$$

Replace trial displacement increment vector with $\triangle \mathbf{u}_{t \rightarrow t+\Delta t}^{\text {corrector }}$, repeat step 2-4.

\subsection{Validation of the Subcycling-Accelerated CPFE Model}

A virtual microstructure of the polycrystalline $\mathrm{Mg}$ alloy AZ31 is simulated to verify the accuracy and reliability of the subcycling algorithm. The statistically equivalent RVE (SERVE) is constructed using the DREAM.3D software (Groeber and Jackson, 2014) following methods described in (Groeber et al., 2008a,b). The dimension of the SERVE is $25 \mu \mathrm{m} \times 25 \mu \mathrm{m} \times 25 \mu \mathrm{m}$, which contains 24 grains discretized into 16371 four-node linear tetrahedral (TET4) elements and 3270 nodes. A constant strain-rate loading of $0.0001 \mathrm{~s}^{-1}$ is imposed on the top surface, while minimum displacement boundary conditions are imposed on other surfaces to just remove the rigid body modes. 12 active slip systems, distributed among three different slip families are considered for plastic deformation. They are the $\langle a\rangle$-basal, $\langle a\rangle$-prismatic and second order $\langle c+a\rangle$ pyramidal slip systems. The constitutive parameters for each slip system are calibrated in (Cheng and Ghosh, 2015) and used in this test. Twin systems are not include in this verification simulation, because additional numerical treatment is required, which will be discussed in section 5.3.

In the subcycling-accelerated CPFE simulations, elements are transitioned from the coarse to the fine time-scale zone if the material constitutive model for that element fails to converge with coarse time-scale integration. At 10\% strain, 2368 elements of the 16371 elements have transitioned into the fine time-scale, which corresponds to $14.15 \%$ of all elements. The volume-averaged stress-strain response by the subcycling-accelerated and conventional CPFE simulations are compared in figure 8a. Excellent agreement is found 
between the two results. To further examine accuracy, the local stress contour plots are compared in figures $8 \mathrm{~b}$ and $8 \mathrm{c}$. The local stress component $\sigma_{x x}$ at $3 \%$ strain are plotted in figure 9 along a line passing through the microstructure. Almost negligible difference is seen in the results by the two CPFE analyses. In terms of efficiency, for $10 \%$ strain simulation, conventional CPFE model takes 285 time steps for a total CPU time of 21250 seconds, whereas subcycling-accelerated simulation takes only 109 steps for a total CPU time of 10182 seconds. This corresponds to almost a $100 \%$ increase in efficiency. The subcycling algorithm is also quite efficient for parallel computing. The separate FE problems for fine and coarse time scale domains are solved simultaneously using all processors, unlike other local time-step refinement methods, in which the processors in time-stepunrefined regions remain idle for periods of the simulation time (Zhang et al., 2014).

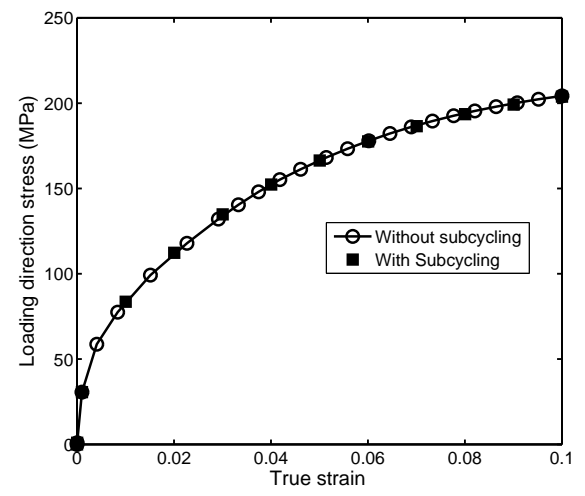

(a)

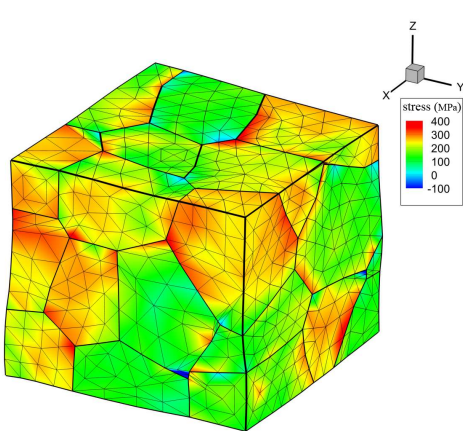

(b)

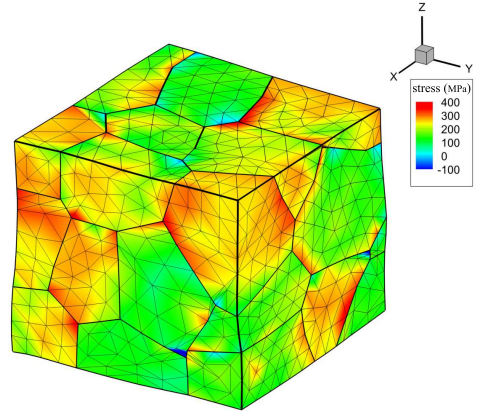

(c)

Figure 8: Comparison of (a) volume-averaged stress-strain response, and loading direction stress $\sigma_{x x}$ distribution in the 24 grain polycrystalline microstructure with (b) with subcycling-accelerated model, and (c) conventional CPFE analysis.

\subsection{Implementation of Subcycling Algorithm for Twinning}

The proposed subcycling algorithm provides an efficient way to model twinning induced heterogeneous deformation by partitioning both the spatial and time domains. Twins undergo a fast shear and are prime candidates to be selected as the fine time-scale domain in the subcycling model. An implicit twin formation algorithm is proposed incorporating both the subcycling method and the CPFE time integration scheme in table 3 . The steps in this algorithm are detailed next. 


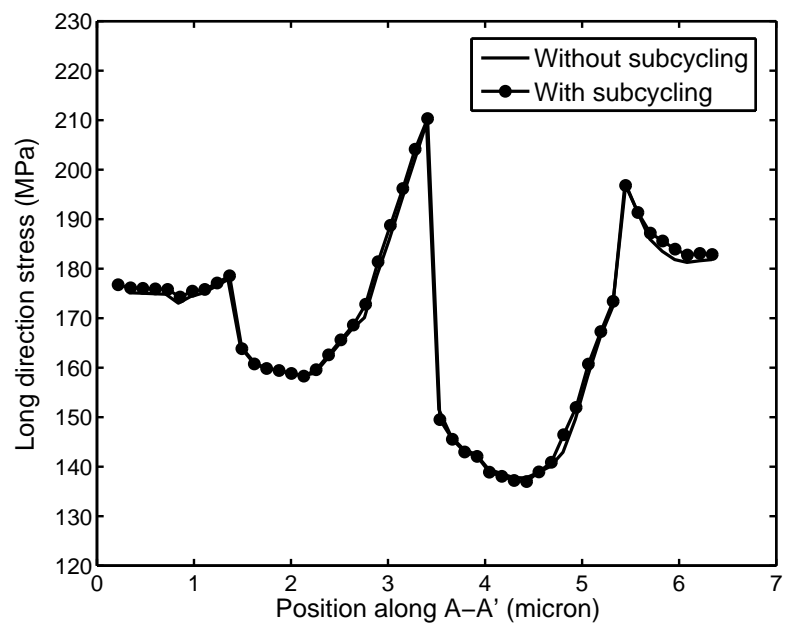

Figure 9: Local stress $\sigma_{z z}$ along a line through the middle section of the 24 grain polycrystalline microstructure by the subcycling-accelerated and conventional CPFE analyses.

For integrating from time $t$ to $t+\Delta t$ with known deformation variables at time $t$, the steps proceed as follows.

1. Assign elements in existing twin bands at time $t$, which are undergoing high strainrates, to the fine time-scale domain. Use the subcycling algorithm to solve for the displacement increments $\triangle \mathbf{u}^{\text {trial }}$ and stress $\sigma^{\text {trial }}$ at time $t+\triangle t$. Update the state and internal variables at $t+\triangle t$, keeping twins fixed in this step.

2. Use the stress $\sigma^{\text {trial }}$ in the twin nucleation criteria of equation (27) and twin propagation criteria of equations (29) and (31) to check for the nucleation of new twins and propagation of existing twins.

3. Update the twinned configuration and add the newly twinned elements to the fine time-scale domain.

4. Return back to time $t$. Use the known state and internal variables at time $t$ and the updated fine time-scale domain to solve for displacements at the time increment $t \rightarrow t+\triangle t$ again. This step ensures that the twin nucleation, propagation, and their induced deformation heterogeneities are captured adequately by using smaller time steps.

In the absence of step 4, the algorithm becomes explicit and staggered in terms of updating twins. This can introduce significant errors due to the fact that highly heterogeneous deformation in the new twins are not captured using fine time steps. 


\subsection{Validation of the Subcycling-Accelerated CPFE Model for Discrete Twin Evolution}

A single crystal simulation of pure $\mathrm{Mg}$ is conducted to validate the accuracy and efficiency of the subcycling-accelerated CPFE model for discrete twin evolution. The microstructure simulated has a dimension of $20 \mu \mathrm{m} \times 10 \mu \mathrm{m} \times 10 \mu \mathrm{m}$. It is discretized into 67418 four-node linear tetrahedral (TET4) elements with 13021 nodes. A uniaxial constant strain-rate $1 \times 10^{-4}$ is applied on the top surface along the $\mathrm{X}$-axis, and minimum displacement boundary conditions are applied to the bottom surface, as shown in figure 10a. The crystal has a orientation that is specified by Euler angles $\left[0^{\circ}, 5^{\circ}, 0^{\circ}\right]$ in the Z-X-Z convention, with the [0001] lattice direction aligned at $5^{\circ}$ from the Z-axis. The loading direction leads to the formation of $\{10 \overline{1} 2\}$ extension twins. The $5^{\circ}$ slight tilting of the crystal orientation makes the Schmid factor of twin variant 1 to be the highest among all six twin variants. Without this tilt, both the twin variants 1 and 4 have equal Schmid factors and the interaction between different twin variants must be considered. This is not accounted for in the current work. To allow for formation of heterogeneous twins, the twin nucleation source is perturbed by a small amount. The length $L$ of the initial sessile dislocation $\langle c+a\rangle$ at each Gauss point is perturbed by random sampling from points on a Weibull distribution. Besides deformation twinning, 12 active dislocation slip systems belonging to three families, viz. the $\langle a\rangle$-basal, $\langle a\rangle$-prismatic and $\langle c+a\rangle$ second order pyramidal slip systems, are responsible for plastic deformation (Graff et al., 2007). Constitutive parameters for pure $\mathrm{Mg}$, calibrated in (Cheng and Ghosh, 2015), are used in these simulations.

The single crystal simulations are conducted using (i) the subcycling-accelerated implicit twin evolution model, and (ii) an explicit staggered algorithm. Various simulations with the explicit staggered algorithm and different time step sizes,e.g. $\triangle t=1,2,4,6,8,10$ seconds are performed to obtain a reference solution. Convergence is studied in figure 10b, a plot of the fraction of the number of twinned elements over the total number of elements at $1 \%$ strain. For time steps exceeding 2 seconds, twins predicted by this method are divergent. Therefore the results with a time step of $\triangle t=1 \mathrm{~s}$ is chosen as the reference solution. The subcycling-accelerated twin formation algorithm in section 5.3 is then validated by conducting a simulation with a starting coarse time-step of 10 seconds. The number fraction of the twinned elements as a function of the applied macroscopic strain is compared with the reference solution in figure 11a. Results by the implicit subcycling model with $\triangle t=10 \mathrm{~s}$ shows very good agreement with the reference solution. However, solutions with larger time steps in the explicit staggered algorithm suffer from accuracy.

Figure $11 \mathrm{~b}$ plots the stress-strain response by different models up to $10 \%$ strain. Prior

to $7 \%$ strain, the stress and hardening rate are very low, since deformation is dominated by twin nucleation and propagation induced plastic flow. Near $7 \%$ strain, twins begin to 
saturate and occupy large volumes in the crystal. The dominant deformation mechanism then switches to $\langle c+a\rangle$ dislocation slip and the hardening rate increases rapidly. This causes the sigmoidal shape of the stress-strain curves, experimentally observed for $\mathrm{Mg}$. All simulations predict this change in deformation mechanisms and the sigmoidal shape of the stress-strain response curves. However, large time step simulations with the explicit staggered twin update scheme, predicts lower hardening rate after the twin saturates. The subcycling model however yields results that are identical to the reference solution.

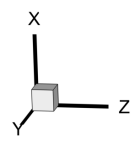

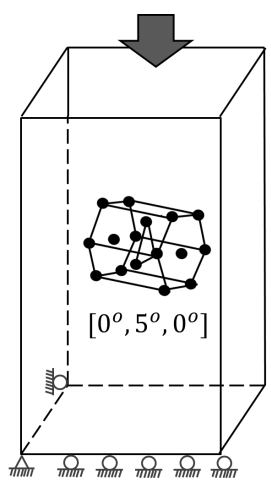

(a)

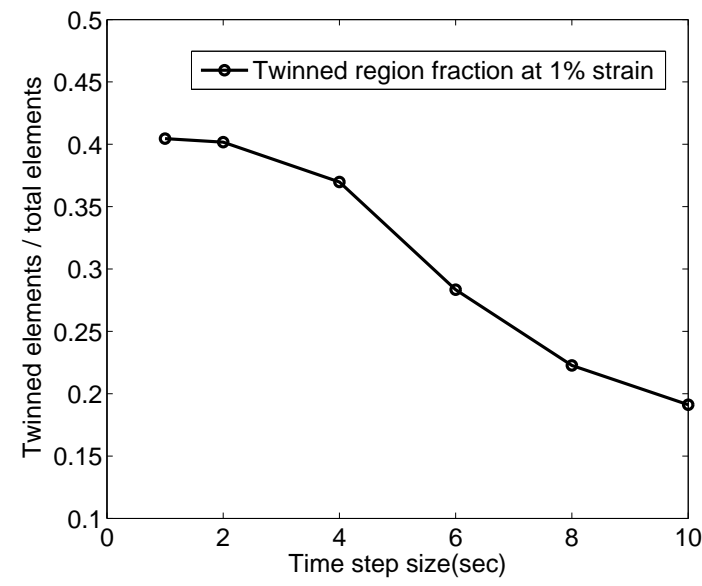

(b)

Figure 10: (a) Schematic of the computational model of single crystal Mg under constant strain-rate uniaxial loading with Schmid factor, and (b) number fraction of twinned elements in the volume as a function of the time-step size.

A comparison of the discretely twinned microstructure predicted by different models is made in figure 12. Figure 13 compares the Lagrangian strain $E_{y y}$ distribution at $1 \%$ strain. The solutions by the small time-step staggered model and the subcycling model are shown in figures $12 \mathrm{a}$ and $13 \mathrm{a}$ respectively. The $\left[0^{\circ}, 5^{\circ}, 0^{\circ}\right]$ orientation (Euler angles) causes the (1102) [1101] twin variant to have the highest Schmid factor in comparison with other extension twin variants. The only exception is that a (1012) [1011] twin variant (variant 4 of the extension twin systems) occurs at the upper left corner of the model due to the local stress-state. This is shown in figures $12 \mathrm{a}$ and $12 \mathrm{c}$ respectively. The subcycling-enhanced CPFE simulation predicts almost the same twinned microstructures as the reference solution by the staggered method with a small time-step. Results of the staggered method with a larger time step $\Delta t=10 \mathrm{~s}$ in figure $12 \mathrm{~b}$ however do not capture the variant 4 twin and also predict a smaller twinned volume fraction. Deformation localization is predicted 


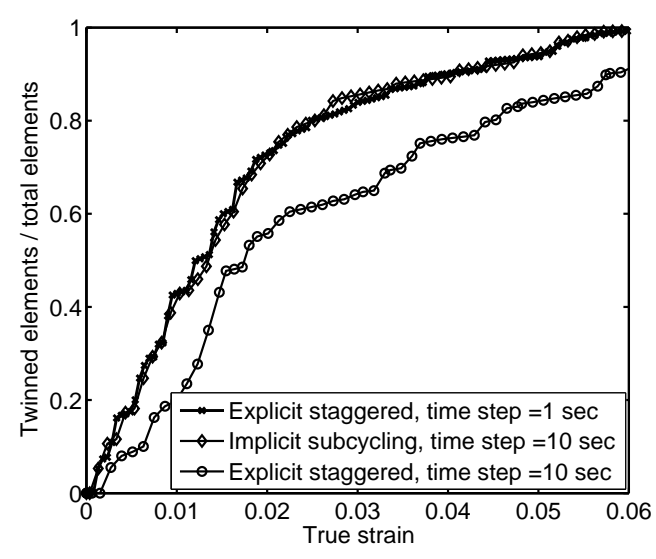

(a)

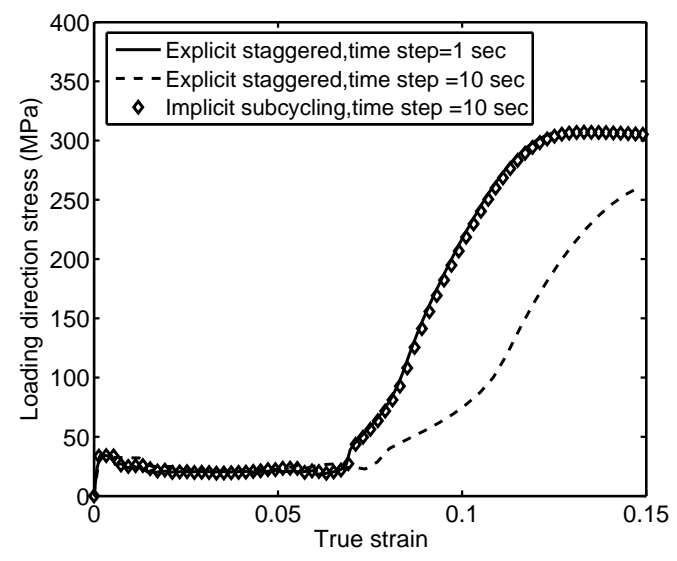

(b)

Figure 11: Comparison of (a) evolution of fraction of twinned elements as a function of strain, and (b) stressstrain response of single crystal Mg simulations by using the explicit staggered and implicit subcycling twin update models.

within twin bands in the reference and subcycling model solutions. Plastic deformation requires gliding on twin systems or dislocation glide in the $\langle c+a\rangle$ slip systems to compress the crystal along the $\mathrm{X}$-axis. The localized strain distribution is caused by easy gliding of micro-twins, in contrast to the $\langle c+a\rangle$ system dislocation glide with high shear resistance.

Significant computational efficiency is achieved with the subcycling model. It takes $1273 \mathrm{CPU}$ seconds to compute up to $10 \%$ strain in comparison with 4576 seconds with the staggered model with a time-step of $\triangle t=1 s$. This corresponds to a 3.6 times acceleration with the subcycling model with high accuracy. In summary, the subcycling-accelerated CPFE model is found to be very effective for modeling deformation leading to discrete twin evolution and is used in the remainder of this paper.

\section{Deformation and Discrete Twin Band Formation in Polycrystalline Mg}

This section studies discrete twin formation and twin-induced heterogeneous deformation in the representative polycrystalline microstructure of high-purity $\mathrm{Mg}$ by using the subcycling-accelerated CPFE model. Results are analyzed and compared with the experiments in (Beyerlein et al., 2011, 2010).

Image-based microstructural volumes simulated with the CPFE model are generated to be statistically equivalent to the experimental microstructures described in (Beyerlein 


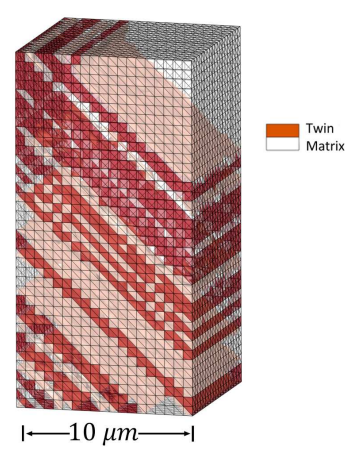

(a)

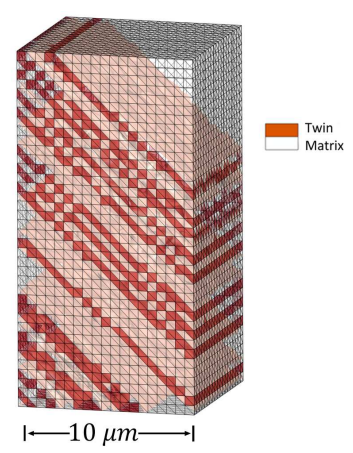

(b)

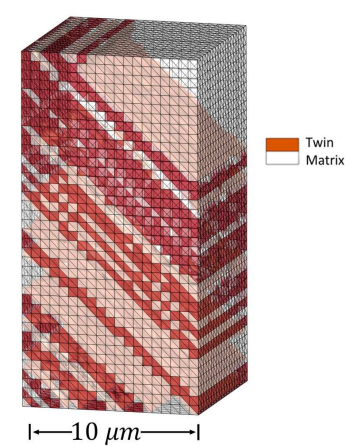

(c)

Figure 12: (a) Simulated twinned single crystal Mg microstructure at $1 \%$ strain, using: (a) explicit staggered model with a time step $\Delta t=1 \mathrm{~s}$, (b) explicit staggered model with a time step $\Delta t=10 \mathrm{~s}$, and (c) implicit subcycling model with a time step $\triangle t=10 \mathrm{~s}$.

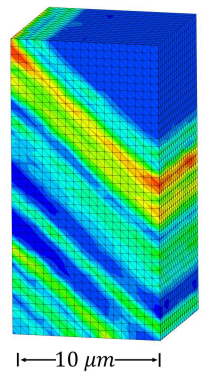

(a)

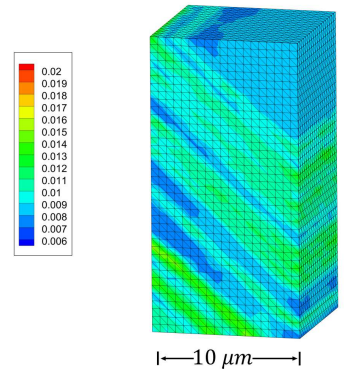

(b)

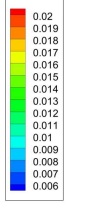

(c)

Figure 13: Lagrangian strain $E_{y y}$ distribution at $1 \%$ overall strain, using: (a) explicit staggered model with a time step $\triangle t=1 \mathrm{~s}$, (b) explicit staggered model with a time step $\triangle t=10 \mathrm{~s}$, and (c) implicit subcycling model with a time step $\triangle t=10 \mathrm{~s}$. 
et al., 2010). Statistical distributions of the grain size, crystallographic orientation and crystallographic misorientation across grain-boundaries are obtained from the experimental results of (Beyerlein et al., 2010). This discrete data is fitted to probability distribution functions for input into the microstructure generator software Dream3D (Groeber and Jackson, 2014). For a specified number of grains in the ensemble, Dream3D generates a statistically equivalent virtual microstructure with statistical distributions, similar to the experimental data. The generated virtual microstructure contains 620 grains with an average grain diameter of $32 \mu \mathrm{m}$. The texture of the virtual microstructure is represented by pole figure plots $14 \mathrm{~b}$. The $300 \mu \mathrm{m} \times 300 \mu \mathrm{m} \times 300 \mu \mathrm{m}$ computational domain is discretized into 520404 four node tetrahedrons consisting of 96849 nodes. A uniaxial, constant strain-rate loading of $1 \times 10^{-3} s^{-1}$ is applied along the Y-axis, which is normal to transverse direction (TD) surface of the virtual microstructure as shown in figure 14a. The color contour corresponds to the angle of alignment between the [0001] lattice axis in each grain and the normal direction (ND). A minimum displacement boundary condition, constraining the rigid body modes, is applied to the bottom surface. The pole figure shows that most grains have their [0001] lattice axis aligned close to ND direction and perpendicular to TD direction. Compression along the TD direction thus causes elongation of grains along the [0001] lattice axis, nucleating $\{10 \overline{1} 2\}$ twins.

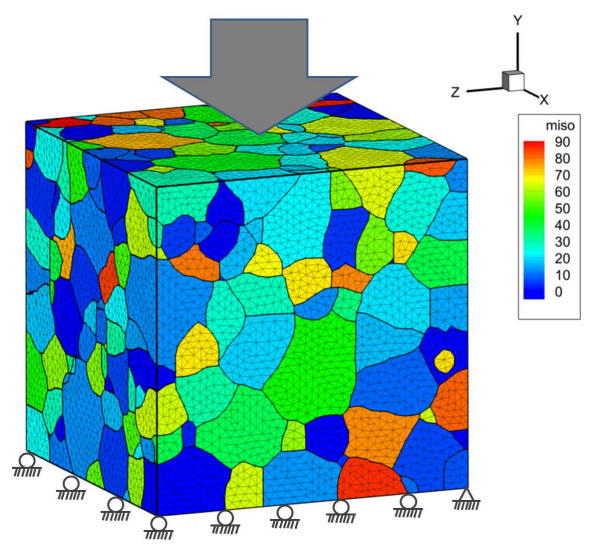

(a)

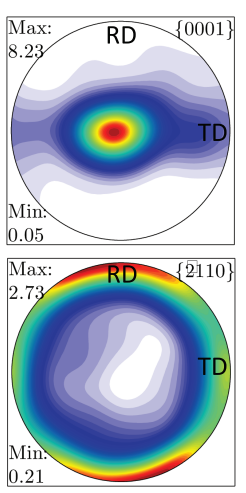

(b)

Figure 14: (a) Computational model of polycrystalline Mg showing boundary conditions and contour plot of the alignment between [0001] lattice direction and loading direction, and (b) pole figures showing the texture of the representative microstructure.

A comparison of computational efficiency of the CPFE models, shows that the conventional model without subcycling takes $172832 \mathrm{CPU}$ seconds, whereas the model with 


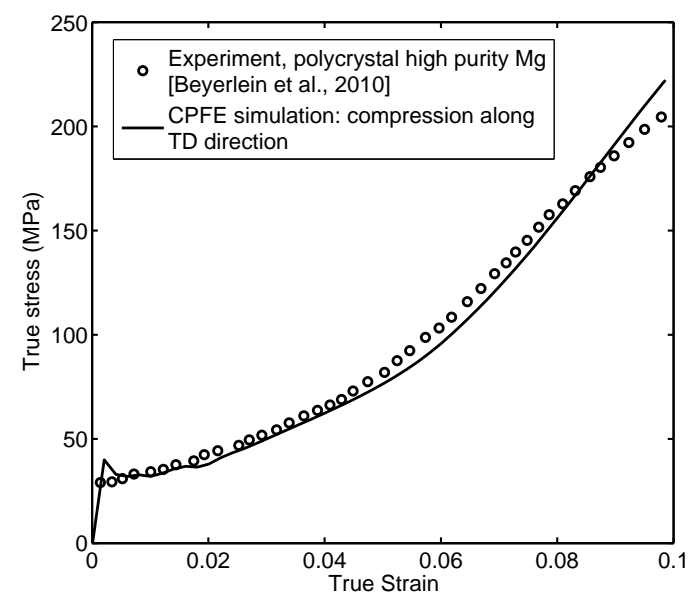

Figure 15: Comparing volume-averaged stress-strain response from simulations with experimental results in (Beyerlein et al., 2011, 2010).

Table 5: Crystal plasticity constitutive parameters for twin evolution in pure $\mathrm{Mg}$.

\begin{tabular}{|c|c|c|c|c|c|}
\hline$s_{t w}(\mathrm{MPa})$ & $\dot{\gamma}_{0, t w}\left(s^{-1}\right)$ & $f_{\text {shuffle }}\left(s^{-1}\right)$ & $\lambda_{\text {shear }}(\mathrm{nm})$ & $d_{\text {tw }}(\mathrm{nm})$ & $\triangle F(\mathrm{~J})$ \\
\hline 12 & 0.0023 & $1 \times 10^{13}$ & 1 & 0.4 & $0.8 \times 10^{-19}$ \\
\hline \hline$P_{\text {promoter }}$ & $\rho_{\text {tot }}\left(m^{-2}\right)$ & $l_{\text {tw }}(\mathrm{nm})$ & $q_{\text {slip-twin }}$ & $q_{\text {twin-slip }}$ & \\
\hline 0.0027 & $1 \times 10^{14}$ & 50 & 1 & 0.001 & \\
\hline
\end{tabular}


subcycling model takes on $29844 \mathrm{CPU}$ seconds to compute up to $2 \%$ strain. This corresponds to a factor of $\sim 6$ speed-up. This efficiency is necessary when modeling twins is a priority.

\subsection{Stress-Strain Behavior}

The constitutive parameters for the twin propagation model in section 3.2 are calibrated from experimental results in (Beyerlein et al., 2010) and are listed in table 5. The CPFE simulations with discrete twin formation model capture the experimentally observed sigmoidal shape stress-strain response of Mg polycrystal very well, as shown in figure 15 . The sigmoidal shape in the stress-strain response is the consequence of the transition from a twin-induced low hardening-rate to a dislocation slip induced high hardening-rate when twins saturate. When a new twin nucleates and starts to propagate, the material undergoes local softening since twin nucleation requires a higher stress than that needed for propagation. The subsequent hardening-rate of twin system resistance is low. The overall hardening-rate of the material remains low when the microstructure undergoes massive twin propagation. This is observed both in simulations in figure 16b, and in experiments (Beyerlein et al., 2010). When most grains have twinned and cannot deform by additional twinning, they start to deform by $\langle c+a\rangle$ dislocation slip. This manifests as an increase in the hardening-rate in the stress-strain response at around $6 \%$ strain, as shown in figure 15. The twin volume fraction $f_{t w}$ in the figure is defined as $f_{t w}=\frac{\sum_{i=1}^{n e l} \int_{\Omega^{i}} \gamma_{t w} d \Omega^{i}}{\gamma_{t w}^{m a x} \Omega^{S E R V E}}$. Tensioncompression asymmetry in the stress-strain response is also predicted in figure 16a. The tension test simulation is conducted along the TD-direction, for which most of the grains along the [0001] lattice axis undergo compression, thus impeding $\{10 \overline{1} 2\}$ twin nucleation. The tension simulation results show much higher yield stress and hardening-rate since only the dislocation slip hardening dominates deformation in most grains.

\subsection{Analysis of Twin Evolution}

Texture evolution in polycrystalline microstructures and texture change due to crystal lattice reorientation caused by twins, are studied in this section. Contour plots of the simulated texture evolution are shown in figures $17 \mathrm{a}$ and $17 \mathrm{~b}$ for $2 \%$ and $6 \%$ strains respectively. In the pre-deformation stage, most grains have their [0001] lattice axes aligned with the ND direction (parallel to Z-axis) as seen in figure 14a. With propagation of a twin band through the grain, the lattice in the twinned region is reorientated by nearly $86^{\circ}$, as shown in figure 17a. At $6 \%$ strain, many grains that originally have their [0001] lattice axis aligned with the ND direction, are dominated by twins and their lattices are reorientated in figure $17 \mathrm{~b}$. On the other hand, grains that have their [0001] lattice axis originally aligned at $30^{\circ}-60^{\circ}$ to the ND direction do not have high twin volume fractions, as they can deform 


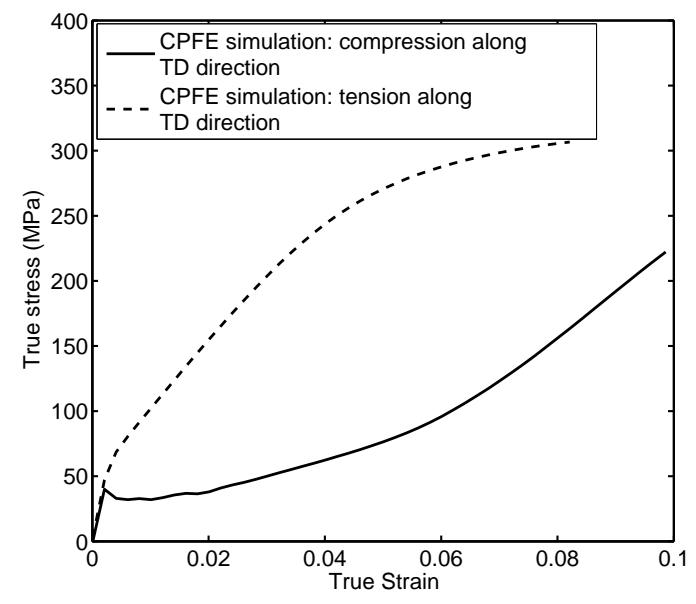

(a)

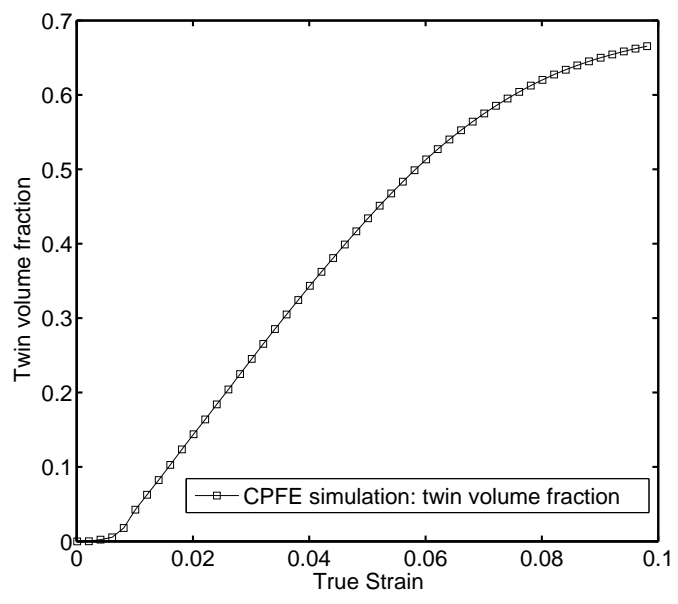

(b)

Figure 16: (a) Volume-averaged stress-strain plots by tension and compression simulations of the polycrystal microstructure loaded in the TD-direction, (b) evolution of the twin volume fraction $f_{t w}$.

by basal slip systems with much smaller shear resistance.

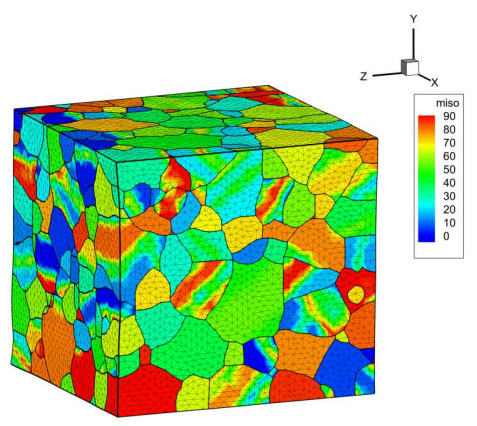

(a)

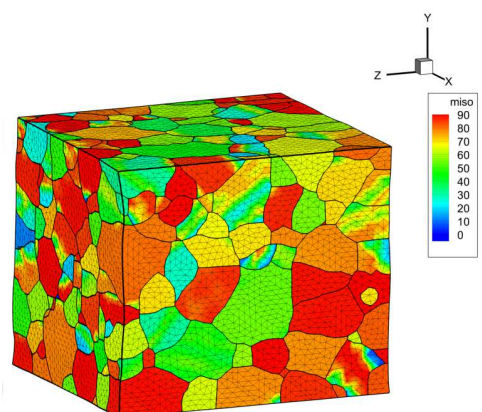

(b)

Figure 17: Texture evolution with deformation in contour plots of the angles between the [0001] lattice axis in each grain and the ND direction (Z-axis) at (a) $2 \%$ strain and (b) $6 \%$ strain.

Contour plots of lattice misorientation depicting the twinned configuration, the loading direction stress $\sigma_{y y}$ and the Lagrangian strain $E_{y y}$ are shown in figures $18 \mathrm{a}, 18 \mathrm{~b}$ and $18 \mathrm{c}$ respectively at $\sim 0.7 \%$ strain. Strain localization is observed in the twin bands of figure 


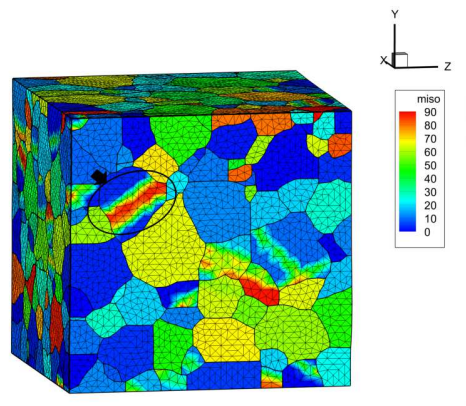

(a)

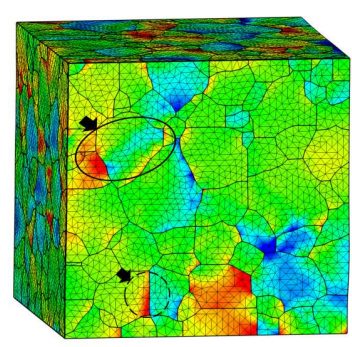

(b)

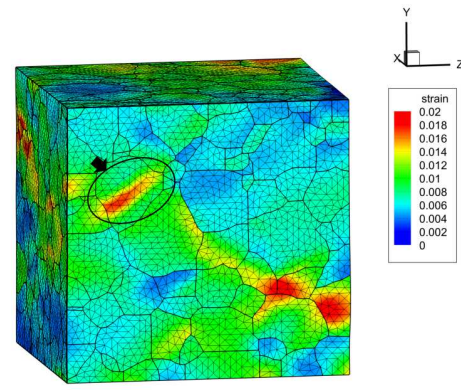

(c)

Figure 18: CPFE simulation predicted contour plots of: (a) polycrystalline microstructure with twins, (b) loading direction stress $\sigma_{y y}$, and (c) loading direction Lagrangian strain $E_{y y}$ at $1 \%$ overall strain. 
$18 \mathrm{c}$ (marked with a black ellipse) with a reoriented crystal lattice of $\sim 86^{\circ}$. Stress concentration is observed in the adjoining grain, close to the intersection of a grain boundary and twin, as circled in figure 18b. This stress concentration is caused by twinning induced accommodation strains, discussed in (Jonas et al., 2011). With twin band propagation and growth, the localized shear strain approaches the grain boundary, which should be accommodated by slip system glide on the adjacent grain. If twin systems in the adjoining grain are aligned with the propagating twins in a grain, the shear strain can be accommodated by nucleating another twin and forming an adjoining grain twin-pair (ATP) (Beyerlein et al., 2011). Otherwise, the twin-induced shear strain must be accommodated by either easy basal slip systems or hard $\langle c+a\rangle$ slip systems, the latter leading to local stress concentrations. Such twin-induced stress concentration can be the primary cause for crack initiation at twin-grain intersections, observed in (Yu et al., 2011).

The sensitivity of twin formation to microstructural features is studied through histograms of the number fraction of twinned grains as functions of the grain size and twin system Schmid factor, respectively in figures 19a and 19b. Results from both experiments in (Beyerlein et al., 2011) and simulations are plotted with satisfactory agreement. Both experimental and simulation results in figure 19a show that small grains are slightly less preferred for twin formations. This is probably due to the fact that they have less number of contiguous grain neighbors and therefore have a lower probability of encountering softhard grain boundaries that often nucleate twins. Most twins tend to nucleate at the hard grain side of a soft-hard grain boundary, as indicated in the statistical analysis of CPFE simulation results of twin nucleation in (Cheng and Ghosh, 2015). Dislocations tend to accumulate in the soft grain side of a typical soft-hard grain boundary, causing stress concentration in the hard grain side with a higher probability of local twin nucleation. Figure 19a shows that grains oriented for high resolved shear stress on twin systems have a higher probability of twin formation. A small number of grains with even negative twin system Schmid factors are found to nucleate twins due to twin-induced nucleation mechanism at grain boundaries. Finally, the histogram of twinned grain boundary number fraction as a function of the [0001] lattice axis misorientation, is plotted in figure 19c. Very small elements in the CPFE analysis, with effective surface areas smaller than $100 \mathrm{\mu m}^{2}$, are not represented in these plots. The simulation results show a noticeable decrease in the probability of twin formation when grain boundary misorientation decreases. A good agreement is observed between the model and experiments.

\section{Concluding Remarks}

An advanced, image-based crystal plasticity FE model, is developed in this paper for predicting discrete twin formation and associated heterogeneous deformation in single 


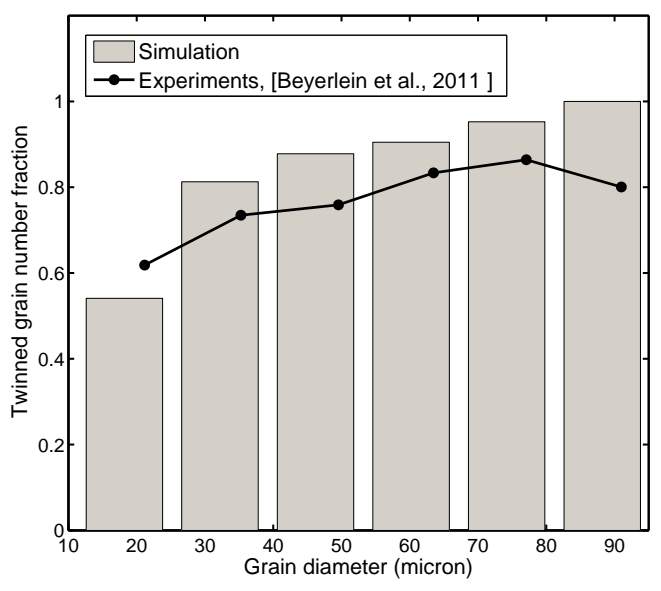

(a)

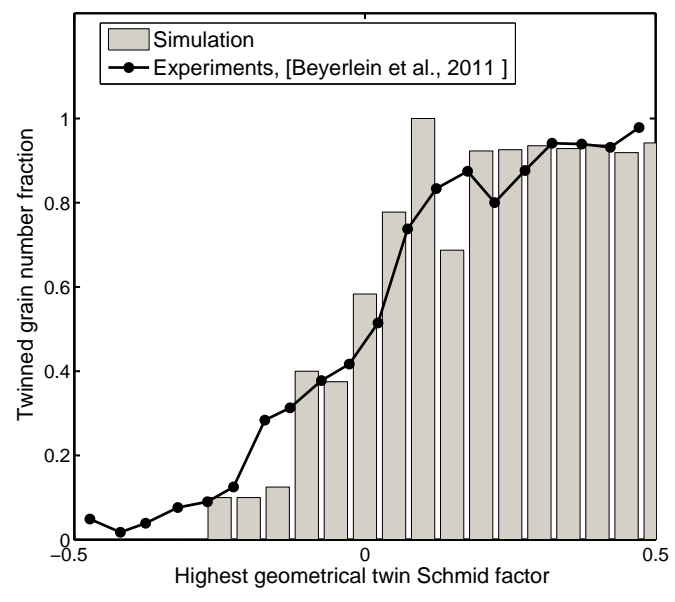

(b)

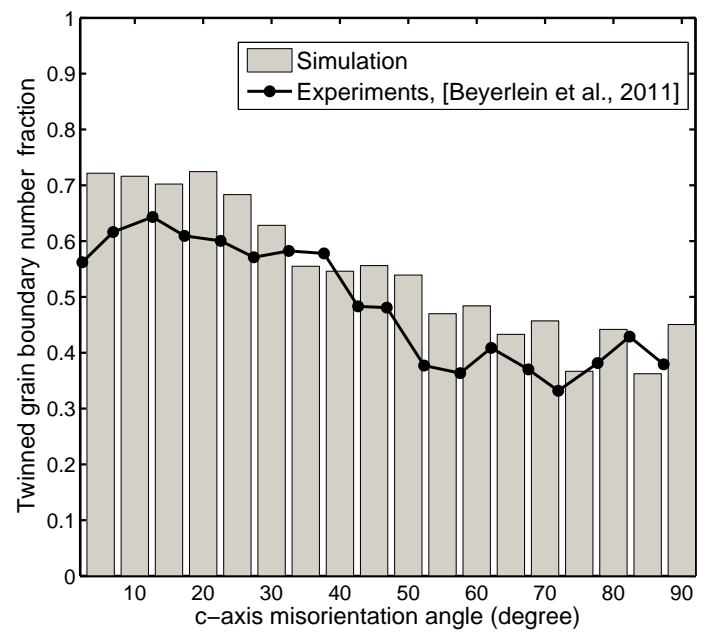

(c)

Figure 19: Histograms of: (a) number fraction of twinned grains as a function of average grain size, (b) number fraction of twinned grains as a function of highest twin Schmid factor, and (c) twinned grain boundary number fractions as a function of c-axis misorientation angle. 
crystal and polycrystalline microstructures of pure Mg and Mg alloys. The model combines advanced methods of computational mechanics and computational material science to achieve this goal. The physics of nucleation, propagation and growth of explicit twins are considered in the CPFE formulation. The twin nucleation model assumes that existing sessile $\langle c+a\rangle$ dislocations dissociate into stable twin loops and leave behind stair-rod dislocations. Numerical criteria for twin nucleation are developed from energetic analysis of the dissociation process using elastic dislocation theory. This infers that a stable twin loop forms when dissociation is spontaneous and the energy after dissociation satisfies the irreversibility and reliability conditions. The twins propagate by atoms shearing on twin planes and shuffling to reduce the thermal activation energy barrier. A thermal activationbased slip model is constructed to account for the rate of the mixed shear-shuffle process. The twins thicken when the twin loop propagates from one atomic layer to an adjacent layer. This is prompted by existing long dislocation poles that penetrate twin planes. The speed of twin propagation along the twin shearing direction and normal to the twin plane are obtained from the thermal activation rate of twin gliding and the rate of encountering a promoter. Non-local criteria governing the numerical prorogation of discrete twin bands are developed for CPFE simulations.

The proposed explicit twin evolution model however has intrinsic issues of low computational efficiency, since the simulation time increment size is bounded by the high deformation-rate inside twin bands. A subcycling algorithm that invokes multi-time scales is developed for accelerating CPFE simulations. The subcycling method decomposes the computational domain into sub-domains of localized twinning with very fine time-steps for numerical integration, and complementary domains of relatively low resolution deformation. Using differential time steps, the two types of sub-domains are solved as two separate explicit CPFE problems, followed by an assembly process of coupling the nodal residual forces to obtain displacement corrections after each coarse time increment. This increases the accuracy of CPFE simulations, while significantly enhancing efficiency. A 6-fold increase in computing speed is obtained for a polycrystalline $\mathrm{Mg}$ microstructure simulation in this paper.

CPFE simulations of high purity $\mathrm{Mg}$ microstructures predicts the experimentally observed sigmoidal shape of the stress-strain curve and the tension-compression asymmetric response. The orientation evolution, caused by twinning is explicitly captured in each grain. Heterogeneous twin formation with strain localization is predicted in the polycrystalline microstructures and stress concentrations are observed at certain locations of twin-grain boundary intersections. This can be potentially responsible for micro-crack initiation in the microstructure. To the knowledge of the authors, this developed capability 
of explicit twin evolution in polycrystalline microstructures of $h c p$ materials is currently lacking in the literature, but is needed for mitigating twin related material failure.

\section{Appendix A: Derivation of Slip System Hardening Models}

Derivation of the hardening models for slip system resistances are given in this section. Three types of resistances are conventionally considered for mobile dislocation glide in crystal plasticity models (Ma et al., 2006). The first type of resistance comes from the stress field of sessile dislocations on the parallel lattice planes. These sessile dislocations repel a mobile dislocation with a resistance force $\mathbf{F}$, expressed as:

$$
\mathbf{F}=\left(\boldsymbol{\sigma}_{P} \mathbf{b}_{m}\right) \times \mathbf{l}_{m}
$$

where $\sigma_{P}$ is the stress field of the parallel dislocations, and $\mathbf{b}_{m}$ and $\mathbf{l}_{m}$ are the Burgers vector and line direction vector of the mobile dislocation respectively. For the mobile dislocation to pass the parallel sessile dislocations, the critical applied resolved shear stress $\tau_{\text {critical }}$ (derived in (Hull and Bacon, 2001)) must satisfy the condition:

$$
\tau_{\text {critical }} b_{m} \geq F_{m}^{\max }
$$

where $F_{m}{ }^{\max }$ is the maximum value of the component of $\mathbf{F}$ along the slip direction, given as:

$$
F_{m}{ }^{\max }=\frac{G b b_{m} c_{1}}{h}
$$

where $c_{1}$ is a material constant depending on the character of the dislocation line. For pure edge dislocation, $c_{1}=\frac{1}{8 \pi(1-\nu)}$, while $c_{1}=\frac{1}{4 \pi}$ for pure screw dislocation. Additionally, $h$ is the distance between the mobile dislocation slip plane and parallel sessile dislocations illustrated in figure 20, $G$ is the shear modulus, $\nu$ is the Poisson's ratio, $b_{m}$ is the magnitude of $\mathbf{b}_{m}$, and $b$ is the magnitude of the Burgers vector of the resistant parallel sessile dislocations. In figure 20, a sessile dislocations with an arbitrary line direction $l^{\beta}$ can be projected onto a parallel component $l^{\beta}\left|\sin \left(\boldsymbol{n}_{0}{ }^{\alpha}, \boldsymbol{l}_{0}{ }^{\beta}\right)\right|$ and a forest component $l^{\beta}\left|\cos \left(\boldsymbol{n}_{0}{ }^{\alpha}, \boldsymbol{l}_{0}{ }^{\beta}\right)\right|$, thus contributing to both thermal and athermal resistances. Assuming that parallel dislocations in the ensemble are distributed evenly with a uniform spacing $L$, results in a single parallel dislocation line within an area of $L^{2}$, giving the relation:

$$
\rho_{P}=\frac{1}{L^{2}}
$$

Combining equations (49) and (50) with $h=L$, gives a relation between $F_{m}{ }^{\max }$ and the density of parallel dislocations $\rho_{P}$ as:

$$
F_{m}{ }^{\max }=c_{1} G b b_{m} \sqrt{\rho_{P}}
$$




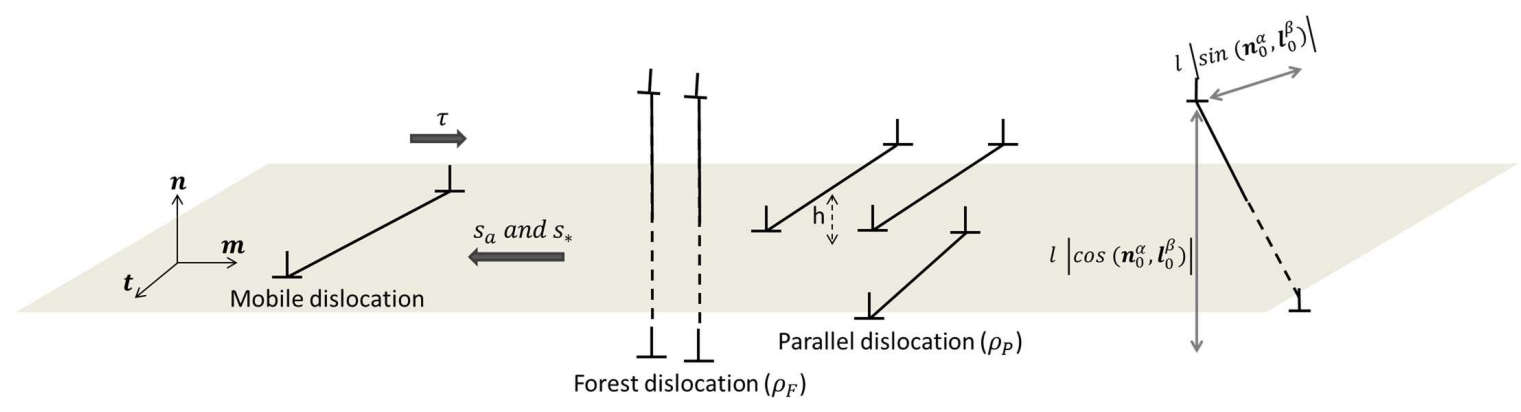

Figure 20: Schematic diagram illustrating the resistance forces on a mobile dislocation, including thermal resistance from forest dislocations and athermal resistance from the stress field of sessile dislocations on parallel planes.

This force causes an athermal resistance to the gliding of mobile dislocations, since it cannot be overcome with the aid of thermal fluctuations. For a slip system $\alpha$, this athermal shear resistance $s_{a, \rho}^{\alpha}$ from long-range stresses of the dislocation structure is equal to the critical resolved shear stress required to overcome $F_{x}{ }^{\max }$. By combining equations (48) and (51), $s_{a, \rho}^{\alpha}$ becomes:

$$
s_{a, \rho}^{\alpha}=\mathrm{c}_{1} G b \sqrt{\rho_{P}^{\alpha}}
$$

where $\rho_{P}^{\alpha}$ is the total density of sessile dislocations that are parallel to the plane of the slip system $\alpha$.

The second type of resistance is from forest dislocations $\rho_{F}$, which act as a short range barrier to mobile dislocation glide. Under low to moderate applied strain-rates, thermal fluctuation is required to overcome the barrier. The rate $R_{\text {cut }}$ of successfully overcoming individual forest dislocation is:

$$
R_{c u t}=\nu_{0} \exp \left(-\frac{\triangle G}{K_{B} \theta}\right)
$$

where $\nu_{0}$ is the attack or attempt frequency and $\triangle G$ is the Gibbs activation free energy, defined as the total free energy barrier minus the mechanical work from the effective applied resolved shear stress $\tau_{e f f}=\tau^{\alpha}-s_{a}^{\alpha}$, expressed as:

$$
\triangle G=Q_{\text {slip }}-\tau_{e f f} V^{*}
$$

The activation volume $V^{*}$ is expressed as:

$$
V^{*}=c_{3} b^{2} \lambda_{\alpha}
$$


where $\lambda_{\alpha}=c_{2} / \sqrt{\rho_{F}}$ is the distance between forest dislocations and $c_{2}, c_{3}$ are material constants. A simplified relation between thermal energy and mechanical work has often been assumed, e.g. in (Hull and Bacon, 2001), as:

$$
\triangle G=Q_{\text {slip }}\left(1-\frac{\tau_{\text {eff }}}{\tau_{\max }}\right)
$$

where $\tau_{\max }=\frac{Q_{s l i p}}{V^{*}}$ is the critical applied shear stress under which, no thermal activation is needed to cut the forest dislocation. The velocity of dislocation gliding is the product of the rate of cutting forest dislocations times the distance between the forest dislocations, i.e. $v=\lambda_{\alpha} R_{c u t}$. Applying this relation to the Orowan equation along with equations (53) and (56), the slip-rate of mobile dislocations is obtained as:

$$
\dot{\gamma}^{\alpha}=\rho_{m} b_{m} v=\rho_{m} b_{m} \lambda_{\alpha} \nu_{0} \exp \left(-\frac{Q_{\text {slip }}}{K_{B} \theta}\left(1-\frac{\tau_{\text {eff }}}{\tau_{\max }}\right)\right)
$$

For moderate rates of plastic deformation corresponding to a high value of $\frac{Q_{s l i p}}{K_{B} T}$, the fraction $\frac{\tau_{e f f}}{\tau_{\max }}$ is of the order $O(1)$. Thus the exponential term $\exp \left(\frac{\tau_{e f f}}{\tau_{\max }}-1\right)$ can be expanded in a Taylor series and only the linear term $\frac{\tau_{e f f}}{\tau_{\max }}$ retained in the relation. In addition, by using the scalar relation $\exp (b a)=(\exp (a))^{b}$, equation (57) can be reduced to a power-law form as:

$$
\dot{\gamma}=\rho_{m} b_{m} \lambda_{\alpha} \nu_{0}\left(\exp \left(\frac{\tau_{\text {eff }}}{\tau_{\max }}-1\right)\right)^{\frac{Q_{s l i p}}{K_{B} T}}=\dot{\gamma}_{0}\left(\frac{\tau_{\text {eff }}}{\tau_{\max }}\right)^{\frac{Q_{s l i p}}{K_{B} T}}
$$

For a reversed loading path, e.g. under fatigue loading conditions, dislocations slip in the opposite direction yielding:

$$
\dot{\gamma}=\dot{\gamma}_{0}\left|\frac{\tau_{\text {eff }}}{\tau_{\max }}\right|^{\frac{1}{m}} \operatorname{sign}\left(\tau_{\text {eff }}\right) \quad \text { with } m=\frac{1}{\frac{Q_{s l i p}}{K_{B} T}}
$$

Clearly, the thermal shear resistance $s_{*, \rho}^{\alpha}$ from cutting the forest dislocations can be defined as the maximum resistance $\tau_{\max }$. From equation (55) and $v=\lambda_{\alpha} R_{c u t}, s_{*, \rho}^{\alpha}$ it is expressed as:

$$
s_{*, \rho}^{\alpha}=\frac{Q_{s l i p} \sqrt{\rho_{F}}}{c_{2} c_{3} b^{2}}
$$

The third type resistance to mobile dislocation glide is from barriers that do not depend on the dislocation based hardening. It includes short range barriers that can be overcome by thermal activation like Peierls resistance, impurities and point defects, as well as long range barriers that are not sensitive to temperature changes, e.g. stress fields from large 
incoherent precipitates or grain boundaries. These resistances are termed as $s_{a, 0}$ and $s_{*, 0}$. They do not evolve with dislocation evolution and serve as the initial resistance. The total athermal and thermal resistances are then the sum of parts related to the dislocation structure and those independent of the dislocation evolution, expressed as:

$$
\begin{gathered}
s_{a}^{\alpha}=s_{a, 0}^{\alpha}+s_{a, \rho}^{\alpha} \\
s_{*}^{\alpha}=s_{*, 0}^{\alpha}+s_{*, \rho}^{\alpha}
\end{gathered}
$$

In general, a dislocation line will not be exactly parallel or normal to the plane of the mobile dislocation on system $\alpha$. In this case, the dislocation line can be projected into two components, viz. the component $l^{\beta}\left|\sin \left(\boldsymbol{n}_{0}{ }^{\alpha}, \boldsymbol{l}_{0}{ }^{\beta}\right)\right|$ perpendicular to slip plane $\alpha$, and the component $l^{\beta}\left|\cos \left(\boldsymbol{n}_{0}{ }^{\alpha}, \boldsymbol{l}_{0}{ }^{\beta}\right)\right|$ parallel to slip plane $\alpha$, as illustrated in figure 20. Here $\mathbf{l}_{\mathbf{0}}^{\beta}$ is the line vector of sessile dislocation on the $\beta$ system in the reference configuration and $l^{\beta}$ is the magnitude or length of the dislocation line. Depending on whether the sessile dislocation on $\beta$ system is edge or screw in the slip plane, the dislocation line vector $\mathbf{l}_{0}^{\beta}$ is in the direction $\mathbf{t}_{\mathbf{0}}\left(=\mathbf{m}_{\mathbf{0}} \times \mathbf{n}_{\mathbf{0}}\right)$ or $\mathbf{m}_{\mathbf{0}}$ respectively. Furthermore, if the sessile dislocation line is normal to its slip plane, e.g. through the climb mechanism, it is an edge dislocation with line vector $\mathbf{l}_{\mathbf{0}}^{\beta}$ in the direction of $\mathbf{n}_{\mathbf{0}}$ (Ma et al., 2006). For simplicity, $\mathbf{l}_{\mathbf{i}} i=1,2,3$ is used to represent all three types of dislocations, with $i=1$ corresponding to screw dislocation, $i=2$ corresponding to in-slip plane edge dislocation and $i=3$ corresponding to the normal-to-slip plane edge dislocation. The parallel and forest dislocation barriers $\rho_{P}^{\alpha}$ and $\rho_{F}^{\alpha}$ that resist mobile dislocations on the $\alpha$ system are thus from all three types of sessile dislocations on all other system. They are expressed as:

$$
\begin{aligned}
& \rho_{P}^{\alpha}=\sum_{\beta} \sum_{i} \chi_{\alpha \beta} \frac{l_{i}^{\beta}}{V}\left|\sin \left(\boldsymbol{n}_{0}^{\alpha}, \boldsymbol{l}_{0, i}^{\beta}\right)\right| \\
& \rho_{F}^{\alpha}=\sum_{\beta} \sum_{i} \chi_{\alpha \beta} \frac{l_{i}^{\beta}}{V}\left|\cos \left(\boldsymbol{n}_{0}^{\alpha}, \boldsymbol{l}_{0, i}^{\beta}\right)\right|
\end{aligned}
$$

where $l_{s}^{\beta}, l_{e t}^{\beta}$ and $l_{e n}^{\beta}$ are the line length of the screw, in-slip-plane edge and normal-to-slip plane edge dislocations, $V$ is the volume of the crystal in the reference configuration and $\chi_{\alpha \beta}$ is a matrix of coefficients that describes the interaction strength between different slip systems. The latter accounts for the self interaction strength, coplanar interaction strength, cross slip strength, glissile junction strength and dislocation lock strength (Ma et al., 2006). For simplicity, all components of $\chi_{\alpha \beta}$ are taken as 1 in this work. The dislocation densities of each type $\rho_{i}$, defined as the dislocation line length per unit volume $\rho_{i} \equiv \frac{\left|\mathbf{I}_{\mathbf{i}}\right|}{V}$, can be further separated into SSDs corresponding to homogeneous plastic deformation, and GNDs 
corresponding to the heterogeneous plastic deformation. The equations (62) can then be rewritten as:

$$
\begin{aligned}
& \rho_{P}^{\alpha}=\rho_{P, S S D}^{\alpha}+\rho_{P, G N D}^{\alpha} \\
& \rho_{F}^{\alpha}=\rho_{F, S S D}^{\alpha}+\rho_{F, G N D}^{\alpha}
\end{aligned}
$$

where

$$
\begin{aligned}
& \rho_{P, S S D}^{\alpha}=\sum_{\beta} \sum_{i} \chi_{\alpha \beta} \rho_{i, S S D}^{\beta}\left|\sin \left(\boldsymbol{n}_{0}^{\alpha}, \boldsymbol{l}_{0, i}^{\beta}\right)\right| \\
& \rho_{P, G N D}^{\alpha}=\sum_{\beta} \sum_{i} \chi_{\alpha \beta} \rho_{i, G N D}^{\beta}\left|\sin \left(\boldsymbol{n}_{0}^{\alpha}, \boldsymbol{l}_{0, i}^{\beta}\right)\right| \\
& \rho_{F, S S D}^{\alpha}=\sum_{\beta} \sum_{i} \chi_{\alpha \beta} \rho_{i, S S D}^{\beta}\left|\cos \left(\boldsymbol{n}_{0}^{\alpha}, \boldsymbol{l}_{0, i}^{\beta}\right)\right| \\
& \rho_{F, G N D}^{\alpha}=\sum_{\beta} \sum_{i} \chi_{\alpha \beta} \rho_{i, G N D}^{\beta}\left|\cos \left(\boldsymbol{n}_{0}^{\alpha}, \boldsymbol{l}_{0, i}^{\beta}\right)\right|
\end{aligned}
$$

are the parallel and forest dislocation densities split into SSDs and GNDs, respectively.

Since the SSDs and GNDs are decomposed linearly, it is convenient to define two types of resistances that are respectively associated with SSDs or GNDs only, as:

$$
\begin{gathered}
s_{a, S S D}^{\alpha}=c_{1} G b \sqrt{\rho_{P, S S D}^{\alpha}} \quad \text { and } s_{a, G N D}^{\alpha}=c_{1} G b \sqrt{\rho_{P, G N D}^{\alpha}} \\
s_{*, S S D}^{\alpha}=\frac{Q_{s l i p}}{c_{2} c_{3} b^{2}} \sqrt{\rho_{F, S S D}^{\alpha}} \quad \text { and } s_{*, G N D}^{\alpha}=\frac{Q_{s l i p}}{c_{2} c_{3} b^{2}} \sqrt{\rho_{F, G N D}^{\alpha}}
\end{gathered}
$$

Combining equations (52), (60) (63) and (65), the total athermal and thermal resistances due to dislocation evolution are related to the SSDs and GNDs components as:

$$
\begin{aligned}
& s_{a, \rho}^{\alpha}=\sqrt{\left(s_{a, S S D}^{\alpha}\right)^{2}+\left(s_{a, G N D}^{\alpha}\right)^{2}} \\
& s_{*, \rho}^{\alpha}=\sqrt{\left(s_{*, S S D}^{\alpha}\right)^{2}+\left(s_{*, G N D}^{\alpha}\right)^{2}}
\end{aligned}
$$

Computation of the SSDs and GNDs are done differently. The material time derivative of $s_{a, S S D}^{\alpha}$ and $s_{*, S S D}^{\alpha}$ are obtained from equations (63), (65) and (66), as well as by assuming that the SSDs considered are only the in-slip-plane edge dislocations. The rates are then given as:

$$
\dot{s}_{a, \rho}^{\alpha}=\frac{c_{1} G b}{2 \sqrt{\rho_{P, S S D}^{\alpha}}} \sum_{\beta} \dot{\rho}_{S S D}^{\beta}\left|\sin \left(\boldsymbol{n}_{0}^{\alpha}, \boldsymbol{t}_{0}^{\beta}\right)\right|
$$




$$
\dot{s}_{*, \rho}^{\alpha}=\frac{Q_{\text {slip }}}{2 c_{2} c_{3} b^{2} \sqrt{\rho_{F, S S D}^{\alpha}}} \sum_{\beta} \dot{\rho}_{S S D}^{\beta}\left|\cos \left(\boldsymbol{n}_{0}^{\alpha}, \boldsymbol{t}_{0}^{\beta}\right)\right|
$$

The evolution rate of SSD densities on slip system $\beta$ slip system is expressed as (Ma et al., 2006):

$$
\dot{\rho}_{S S D}^{\beta}=c_{4} \sqrt{\rho_{F}^{\beta}} \dot{\gamma}^{\beta}+c_{6} d_{\text {dipole }}^{\beta} \rho_{M}^{\beta} \dot{\gamma}^{\beta}-c_{5} \rho_{S S D}^{\beta} \dot{\gamma}^{\beta}-c_{7} \exp \left(-\frac{Q_{b u l k}}{K_{B} \theta}\right) \frac{\left|\tau^{\beta}\right|}{K_{B} \theta}\left(\rho_{S S D}^{\beta}\right)^{2}
$$

where terms on right hand side are due to immobilization of mobile dislocations, dislocation dipole formation, non-thermal annihilation, and thermally activated annihilation, respectively. $c_{4}, c_{5}, c_{6}, c_{7}$, and $c_{8}$ are material constants, $d_{\text {dipole }}$ is the critical distance for dipole formation and $Q_{b u l k}$ is the activation energy for self diffusion. Applying the SSD evolution equation (68) and neglecting thermally activated dislocation annihilation at room temperature, the hardening equations (67a) and (67b) are written as:

$$
\begin{aligned}
& \dot{s}_{a, S S D}^{\alpha}=h_{a}^{\alpha \beta}\left|\dot{\gamma}^{\beta} \sin \left(\boldsymbol{n}_{0}^{\alpha}, \boldsymbol{t}_{0}{ }^{\beta}\right)\right| \\
& \dot{s}_{*, S S D}^{\alpha}=h_{*}^{\alpha \beta}\left|\dot{\gamma}^{\beta} \cos \left(\boldsymbol{n}_{0}^{\alpha}, \boldsymbol{t}_{0}{ }^{\beta}\right)\right|
\end{aligned}
$$

Here $h_{a}^{\alpha \beta}=\frac{c_{1} G b}{\sqrt{\rho_{P}^{\alpha}}} a_{1}$ and $h_{*}^{\alpha \beta}=\frac{Q_{s l i p}}{c_{2} c_{3} b^{2} \sqrt{\rho_{F}^{\alpha}}} a_{1}$, in which $a_{1}=c_{4} \sqrt{\rho_{F}^{\beta}}+c_{6} d_{\text {dipole }}^{\beta} \rho_{M}^{\beta}-c_{5} \rho_{S S D}^{\beta}$. For $h c p$ crystals, the physics-based parameters in $h_{a}^{\alpha \beta}$ and $h_{*}^{\alpha \beta}$ are not clearly identified. In such cases, the phenomenological hardening models can be used to fit hardening rates $h_{a}^{\alpha \beta}$ and $h_{*}^{\alpha \beta}$, expressed as:

$$
\begin{aligned}
& h_{a}^{\alpha \beta}=q^{\alpha \beta} h_{a}^{\beta}=q^{\alpha \beta} h_{a, r e f}^{\beta}\left|1-\frac{s_{a, S S D}^{\beta}}{s_{a, s a t}^{\beta}}\right|^{r} \operatorname{sign}\left(1-\frac{s_{a, S S D}^{\beta}}{s_{a, s a t}^{\beta}}\right) \\
& h_{*}^{\alpha \beta}=q^{\alpha \beta} h_{*}^{\beta}=q^{\alpha \beta} h_{*, r e f}^{\beta}\left|1-\frac{s_{*, S S D}^{\beta}}{s_{*, s a t}^{\beta}}\right|^{r} \operatorname{sign}\left(1-\frac{s_{*, S S D}^{\beta}}{s_{*, s a t}^{\beta}}\right)
\end{aligned}
$$

where $q^{\alpha \beta}$ is a matrix describing latent hardening.

\section{ACKNOWLEDGMENTS}

This work has been supported by a GOALI research program sponsored by the National Science Foundation, Mechanics and Structure of Materials Program through Grant No. CMMI-1100818 (Program Manager: Dr. Kara Peters). The authors gratefully acknowledge this support. They thank their GOALI partner General Motors R\&D for their support of this research. Computing support by the Homewood High Performance Compute Cluster (HHPC) and Maryland Advanced Research Computing Center (MARCC) is gratefully acknowledged. 


\section{References}

Abdolvand, H., Daymond, M.R., 2013a. Multi-scale modeling and experimental study of twin inception and propagation in hexagonal close-packed materials using a crystal plasticity finite element approach-part i: average behavior. J. Mech. Phys. Solids 61, 783-802.

Abdolvand, H., Daymond, M.R., 2013b. Multi-scale modeling and experimental study of twin inception and propagation in hexagonal close-packed materials using a crystal plasticity finite element approach; part ii: Local behavior. J. Mech. Phys. Solids 61, 803 $-818$.

Anahid, M., Samal, M., Ghosh, S., 2011. Dwell fatigue crack nucleation model based on crystal plasticity finite element simulations of polycrystalline Titanium alloys. Jour. Mech. Phys. Solids 59, 2157-2176.

Ardeljan, M., McCabe, R., Beyerlein, I., Knezevic, M., 2015. Explicit incorporation of deformation twins into crystal plasticity finite element models. Comput. Meth. App. Mech. Eng. 295, 396 - 413.

Asaro, R., Needleman, A., 1985. Texture development and strain hardening in rate dependent polycrystals. Acta Mater. 33, 923-953.

Ashby, M.F., 1970. Deformation of plastically non-homogeneous maaterials. Philos. Mag. 21, 399-424.

Balasubramanian, S., 1998. Polycrystalline Plasticity: Application to Deformation Processing of Lightweight Metals. Ph.D. thesis. Massachusetts Institute of Technology.

Barnett, L., 2007. Twinning and the ductility of magnesium alloys: Part i: Tension twins. Mater. Sci. Engng. A464, 1-7.

Belytschko, T., Mullen, R., 1977. Explicit integration of structural problems. Finite Elements in Nonlinear Mechanics 2, 697-720.

Bettles, C., Gibson, M., 2005. Material rate dependence and localized deformation in crystalline solids. Jour. Miner. Met. Mater. Soc. 57, 46-49.

Beyerlein, I.J., Capolungo, L., Marshall, P.E., McCabe, R.J., Tome, C.N., 2010. Statistical analyses of deformation twinning in magnesium. Philos. Mag. 90, 2161-2190. 
Beyerlein, I.J., McCabe, R.J., Tome, C.N., 2011. Effect of microstructure on the nucleation of deformation twins in polycrystalline high-purity magnesium: A multi-scale modeling study. J. Mech. Phys. Solids 59, 988-1003.

Busso, E., Meissonier, F., ODowd, N., 2000. Gradient-dependent deformation of twophase single crystals. Jour. Mech. Phys. Solid. 48, 2333-2361.

Cao, G., Fu, L., Lin, J., Zhang, Y., Chen, C., 2000. The relationships of microstructure and properties of a fully lamellar $T i-A l$ alloy. Intermetallics 8, 647-653.

Cermelli, P., Gurtin, M., 2001. On the characterization of geometrically necessary dislocations in finite plasticity. J. Mech. Phys. Solids 49, 1539-1568.

Cheng, J., Ghosh, S., 2015. A crystal plasticity fe model for deformation with twin nucleation in magnesium alloys. Int. J. Plast. 67, $148-170$.

Cheng, J., Shahba, A., Ghosh, S., 2016. Stabilized tetrahedral elements for crystal plasticity finite element analysis overcoming volumetric locking. Comput. Mech. 57, 733753.

Dai, H., 1997. Geometrically-necessary dislocation density in continuum plasticity theory,FEM implementation and applications. Ph.D. thesis. Massachusetts Institute of Technology.

Deka, D., Joseph, D., Ghosh, S., Mills, M., 2006. Crystal plasticity modeling of deformation and creep in polycrystalline Ti-6242. Metall. Trans. A. 37A(5), 1371-1388.

Van der Giessen, E., Neale, K., 1993. Analysis of the inverse swift effect using a ratesensitive polycrystal model. Computer Methods in Applied Mechanics and Engineering $103,291-313$.

Graff, S., Brocks, W., Steglich, D., 2007. Yielding of magnesium: From single crystal to polycrystalline aggregates. Int. J. Plast. 23, 1957-1978.

Groeber, M., Ghosh, S., Uchic, M.D., Dimiduk, D.M., 2008a. A framework for automated analysis and simulation of 3d polycrystalline microstructures.: Part 1: Statistical characterization. Acta Mater. 56, 1257-1273.

Groeber, M., Ghosh, S., Uchic, M.D., Dimiduk, D.M., 2008b. A framework for automated analysis and simulation of 3d polycrystalline microstructures. part 2: Synthetic structure generation. Acta Mater. 56, 1274-1287. 
Groeber, M.A., Jackson, M.A., 2014. Dream.3d: A digital representation environment for the analysis of microstructure in 3d. Integrating Materials and Manufacturing Innovation 3,5 .

Gurtin, M., 2006. The burgers vector and the flow of screw and edge dislocations in finite-deformation single-crystal plasticity. J. Mech. Phys. Solids 54, 1882-1898.

Gurtin, M., Anand, L., 2009. Thermodynamics applied to gradient theories involving the accumulated plastic strain: The theories of aifantis and fleck and hutchinson and their generalization. J. Mech. Phys. Solids 57, 405-421.

Gurtin, M., Fried, E., Anand, L., 2010. The mechanics and thermodynamics of continua. New York: Cambridge University Press.

Hasija, V., Ghosh, S., Mills, M., Joseph, D., 2003. Modeling deformation and creep in Ti-6Al alloys with experimental validation. Acta Mater. 51, 4533-4549.

Hirth, J.P., Lothe, J., 1982. Theory of Dislocations. 2nd ed., Wiley-Interscience.

Hong, S., Park, S., Lee, C., 2010. Role of $\{10 \overline{1} 2\}$ twinning characteristics in the deformation behavior of a polycrystalline magnesium alloy. Acta Mater. 58, 58735885.

Hull, D., Bacon, D.J., 2001. Introduction to Dislocations. 4th ed., ButterworthHeinemann.

Izadbakhsh, A., Inal, K., Mishra, R.K., 2012. Crystal plasticity based finite element modelling of large strain deformation in am30 magnesium alloy. Model. Simul. Mater. Sci. Eng. 20, 035016.

Izadbakhsh, A., Inal, K., Mishra, R.K., Niewczas, M., 2011. New crystal plasticity constitutive model for large strain deformation in single crystals of magnesium. Model. Simul. Mater. Sci. Eng. 50, 2185-2202.

Jonas, J., Mu, S., Al-Samman, T., Gottstein, G., Jiang, L., Martin, ., 2011. The role of strain accommodation during the variant selection of primary twins in magnesium. Acta Mater. 59, 2046 - 2056.

Kainer, K., 2003. Magnesium alloys and their applications. Wiley-VCH, Weinheim.

Keshavarz, S., Ghosh, S., 2013. Multi-scale crystal plasticity fem approach to modeling nickel based superalloys. Acta Mater. 61, 6549-6561. 
Kocks, U., Argon, A., Ashby, M., 1975. Thermodynamics and kinetics of slip. Progress in materials science $19,141-145$.

Kuchnick, S., Cuitino1, A.M., Radovitzky, R., 2006. Efficient and robust constitutive integrators for single-crystal plasticity modeling. Int. J. Plasticity 22, $1988-2011$.

Li, H., Yang, H., 2012. An efficient parallel-operational explicit algorithm for taylor-type model of rate dependent crystal plasticity. Comput. Mater. Sci. 54, 255 - 265.

Li, Z., Luo, A., Wang, Q., Peng, L., Fu, P., Wu, G., 2013. Effects of grain size and heat treatment on the tensile properties of $\mathrm{mg} 3 \mathrm{nd} 0.2 \mathrm{zn}(\mathrm{wt})$ magnesium alloys. Mater. Sci. Engng. A 564, 450-460.

Ling, X., Horstemeyer, M.F., Potirniche, G., 2005. On the numerical implementation of 3d rate-dependent single crystal plasticity formulations. Int. J. Numer. Meth. Eng. 63, $548-568$.

Ma, A., Roters, F., Raabe, D., 2006. A dislocation density based constitutive model for crystal plasticity fem including geometrically necessary dislocations. Acta Mater. 54, 2169-2179.

Majkut, M., 2013. A study of deformation twinning in magnesium alloy AZ31B. Ph.D. thesis. Queen's University.

Mareau, C., Daymond, M.R., 2016. Micromechanical modeling of twinning in polycrystalline materials: Application to magnesium. Int. J. Plast 85, 803-818.

Neal, M., Belytschko, T., 1989. Explicit-explicit subcycling with non-integer time step ratios for structural dynamics systems. Comput. Struct. 31, 871-880.

Niewczas, M., 2010. Lattice correspondence during twinning in hexagonal close-packed crystals. Acta Mater. 58, 5848-5857.

Rossiter, J., Brahme, A., Simha, M., Inal, K., Mishra, R., 2010. A new crystal plasticity scheme for explicit time integration codes to simulate deformation in $3 \mathrm{~d}$ microstructures: Effects of strain path, strain rate and thermal softening on localized deformation in the aluminum alloy 5754 during simple shear. Int. J. of Plast. 26, 1702 - 1725.

Roters, F., Eisenlohr, P., Bieler, T., 2010a. Crystal plasticity finite element methods in materials science and engineering. Wiley-VCH Verlag $\mathrm{GmbH}$. 
Roters, F., Eisenlohr, P., Hantcherli, L., Tjahjantoa, D., Bieler, T., Raabe, D., 2010 b. Overview of constitutive laws, kinematics, homogenization and multiscale methods in crystal plasticity finite-element modeling: Theory, experiments, applications. Acta Mater. 58, 1152-1211.

Semiatin, S., Bieler, T., 2001. The effect of alpha platelet thickness on plastic flow during hot working of Ti6Al4V with a transformed microstructure. Acta Mater. 49, 35653573.

Serra, A., Pond, R.C., Bacon, D.J., 1991. Computer simulation of the structure and mobility of twinning dislocations in hep metals. Acta Metll. Mater. 39, 1469.

Sloan, S., 1987. Substepping schemes for the numerical integration of elastoplastic stressstrain relations. Int. J. Numer. Meth. Eng. 24, 893911.

Smolinski, P., 1996. Subcycling integration with non-integer time steps for structural dynamics problems. Computers and Structures 59, $273-281$.

Smolinski, P., Belytschko, T., Neal, M., 1988. Multi-time step integration using nodal partitioning. Int. J. Numer. Methods Engrg. 26, 349-359.

Smolinski, P., Wu, Y., 1998. Stability of explicit subcycling time integration with linear interpolation for first-order finite element semidiscretizations. Comput. Meth. App. Mech. Eng. 151, $311-324$. Containing papers presented at the Symposium on Advances in Computational Mechanics.

Staroselsky, A., Anand, L., 2003a. A constitutive model for hcp materials deforming by slip and twinning: application to magnesium alloy $\{$ AZ31B $\}$. Int. J. Plast. 19, $1843-$ 1864.

Staroselsky, A., Anand, L., 2003b. A constitutive model for hcp materials deforming by slip and twinning: application to magnesium alloy az31b. Int. J. Plast. 19, 843-1864.

Venkataramani, G., Ghosh, S., Mills, M., 2007. A size dependent crystal plasticity finite element model for creep and load-shedding in polycrystalline Titanium alloys. Acta Mater. 55, 3971-3986.

Wang, F., Agnew, S., 2015. Dislocation-twin interactions in magnesium alloy az31. Magnesium Technology 2015, 139-144.

Wang, J., Hirth, J.P.and Tom, C., 2009. (1012) twinning nucleation mechanisms in hexagonal-close-packed crystals. Acta Mater. 57, 5521-5530. 
Wong, S., Madivala, M., Prahl, U., Roters, F., Raabe, D., 2016. A crystal plasticity model for twinning- and transformation-induced plasticity. Acta Mater. 118, 140-151.

Yu, Q., Shan, Z., Ju, L., Huang, X., Xiao, L., Sun, J., Ma, E., 2010. Strong crystal size effect on deformation twinning. Nature Letters 463, 08692.

Yu, Q., Zhang, J., Jiang, Y., 2011. Fatigue damage development in pure polycrystalline magnesium under cyclic tensioncompression loading. Materials Science and Engineering: A $528,7816-7826$.

Zambaldi, C., Roters, F., Raabe, D., Glatzel, U., 2007. Modeling and experiments on the indentation deformation and recrystallization of a single-crystal nickel-base superalloy. Mater. Sci. Engng. A 454455, 433440.

Zhang, J., Joshi, S.P., 2012. Phenomenological crystal plasticity modeling and detailed micromechanical investigations of pure magnesium. J. Mech. Phys. Solids 60, 945972.

Zhang, K., Hopperstad, O.S., Holmedal, B., Dumoulin, S., 2014. A robust and efficient substepping scheme for the explicit numerical integration of a rate-dependent crystal plasticity model. Int. J. Numer. Meth. Eng. 99, 239-262.

Zienkiewicz, O., Zhu, J., 1992. The superconvergent patch recovery (spr) and adaptive finite element refinement. Comput.Method Appl M 101, 207 - 224. 